\title{
Die lexikografische Behandlung von Neologismen aus der Perspektive hispanophoner DaF-Lernender
}

Vanessa González Ribao, Leibnitz-Institut für Deutsche Sprache, Mannheim,Deutschland (ribao@ids-mannheim.de)

\begin{abstract}
Zusammenfassung: Anhand von einigen medialen Kommunikationsverben wie mailen oder twittern wird das lexikografische Informationsangebot zu Neologismen auf seine Adäquatheit für die fremdsprachige Produktion untersucht. Die Untersuchung erfolgt aus der Perspektive eines spanischsprachigen DaF-Lernenden. Zur Analyse werden sowohl Neologismenwörterbücher und -datenbanken für das Deutsche als auch gängige, bilinguale Online-Wörterbücher für das Sprachenpaar Spanisch-Deutsch gezogen. Die Ergebnisse der lexikografischen Untersuchung werden exemplarisch mit korpusbasierten Daten aus einer Doktorarbeit verglichen. Die Befunde zeigen den Bedarf und die Notwendigkeit auf, die lexikografische Behandlung von (verbalen) Neologismen im spanisch-deutschen Kontext zu optimieren. Dabei soll — insbesondere - die fremdsprachige Textproduktion berücksichtigt werden.
\end{abstract}

Stichwörter: KOMMUNIKATIONSVERBEN, NEOLOGISMENLEXIKOGRAFIE, ONLINELEXIKOGRAFIE, BILINGUALE WÖRTERBÜCHER, SPANISCH-DEUTSCH, FREMDSPRACHIGE TEXTPRODUKTION, SPANISCHSPRACHIGE DAF-LERNENDER

\begin{abstract}
The Lexicographic Treatment of Neologisms from the Perspective of Hispanophone Learners of GFL. On the basis of some instrumental verbs of communication such as mailen (to mail) or twittern (to tweet) we examined the lexicological information offered on neologisms for its adequacy for text production in a foreign language. The study is carried out from the perspective of a Hispanophone learner of GFL. We analyzed neologism dictionaries and databases for German as well as bilingual online dictionaries for Spanish-German. We compared the results of the lexicographic investigation with corpus-based data from a doctoral thesis. The findings point out the need and necessity of improving the treatment of (verbal) neologisms in Spanish-German dictionaries. When doing so the text production in a foreign language should be particularly considered.
\end{abstract}

Keywords: VERBS OF COMMUNICATION, NEOLOGISM LEXICOGRAPHY, ONLINE LEXICOGRAPHY, BILINGUAL DICTIONARIES, SPANISH-GERMAN, TEXT PRODUCTION IN A FOREIGN LANGUAGE, HISPANOPHONE LEARNERS OF GFL 


\section{Einleitung}

Die vorliegende lexikografische Untersuchung findet im Rahmen einer Dissertationsarbeit zum Thema Argumentstruktur (AS) und Bedeutung medialer Kommunikationsverben (MKV) des Deutschen und des Spanischen im Sprachvergleich statt (González Ribao 2019). Als MKV werden diejenigen Kommunikationsverben bezeichnet, die sich auf Sprachhandlungen beziehen, in denen die Kommunikation zwischen Sprecher und Hörer mithilfe eines technologischen Mediums (d.h. eines technischen Geräts bzw. über das Internet mithilfe einer Anwendungssoftware oder Webseite) erfolgt. Die in dieser Studie analysierten MKV werden in der Abbildung 1 aufgeführt. Ausgangspunkt der lexikografischen Untersuchung sind einschlägige lexikografische Studien für das betreffende Sprachenpaar, die gezeigt haben, dass v.a. bei Produktionssituationen mehr syntagmatische Daten als die in den herkömmlichen Wörterbüchern angeboten werden, notwendig sind (vgl. Meliss 2013, 2015a, 2015b, 2016). Da die Gruppe der MKV sowohl im Deutschen als auch im Spanischen zum größeren Teil aus Neologismen besteht (siehe Abb. 1), stehen diese neuen Verben im Mittelpunkt der Untersuchung. Das Ziel ist zu überprüfen, ob hispanophone DaF-lernende die notwendigen Informationen zu Neologismen für die Textproduktion im Deutschen finden. Daher stehen im Fokus der Untersuchung sowohl die deutschsprachige Neologismenlexikografie (vgl. Lemnitzer 2010) als auch die bilinguale Lernerlexikografie im spanisch-deutschen Kontext. Der Begriff „Lernerlexikografie" ist relativ umstritten (vgl. Tarp 2008: 125-130, Engelberg und Lemnitzer 2009: 27-29). Ein Lernerwörterbuch wird hier als ein Wörterbuch definiert, das auf die Bedürfnisse eines Benutzers (vgl. Tarp 2013b: 463-465, Tarp 2008: 56-58) beim Spracherwerb ausgerichtet ist. Zur bilingualen Lernerlexikografie gehören demnach zweisprachige Wörterbücher, die für die Hilfestellung beim Erwerbsprozess einer Fremdsprache konzipiert sind (vgl. Tarp 2013a: 425-426). Als schriftliche und mündliche Produktion in der Fremdsprache zählen normalerweise folgende Situationen: Texterstellung, (Hin-)Übersetzung, Textbe- und überarbeitung sowie Textwiedergabe (vgl. Tarp 2008: 59-68 und 149-161, Engelberg und Lemnitzer 2009: 112-113 und 120-131, Wiegand et al. 2017: 341-342). Die Ergebnisse der vorliegenden Untersuchung zeigen die Notwendigkeit lexikografischer Ressourcen zu Neologismen für fremdsprachige Produktionszwecke aus der Perspektive spanischsprachiger DaFLernender auf. 


- telegrafieren
- morsen
- telefonieren
- anrufen
- faxen
- simsen
- funken
- übertragen
- ausstrahlen
- senden
- facebooken
- twittern
- posten
- chatten
- bloggen
- mailen
- skypen
- whatsappen

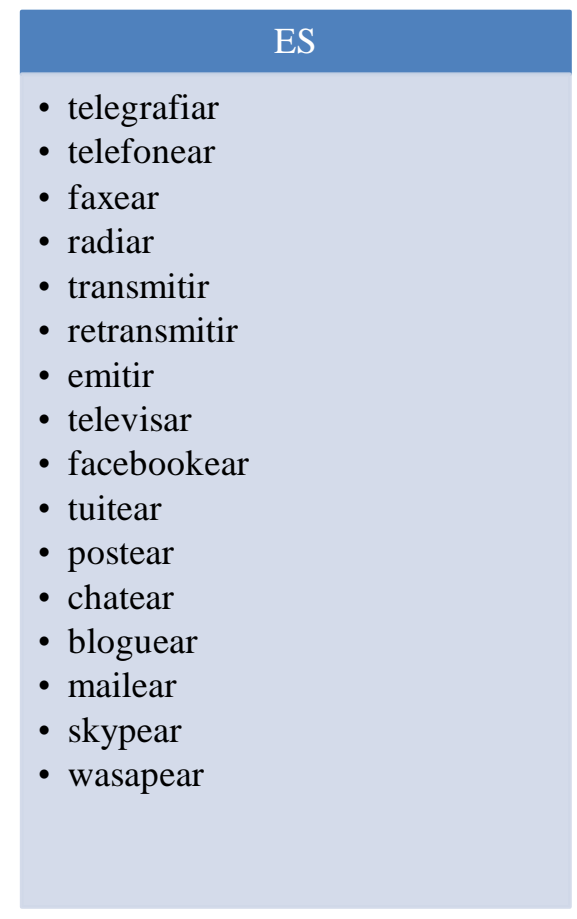

Abb. 1: Die in der Doktorarbeit analysierten MKV des Deutschen und des Spanischen

Der Beitrag ist folgendermaßen aufgebaut: Zunächst wird die angewendete Methodologie kurz präsentiert (siehe Abschnitt 2). Eine Bestandsaufnahme und eine Analyse gängiger lexikografischer Ressourcen zu Neologismen im Deutschen werden in den Abschnitten 3 und 4 durchgeführt. Danach folgt eine Sichtung der Aufführung von Neologismen in einigen ausgewählten bilingualen Wörterbüchern für das betreffende Sprachenpaar Spanisch-Deutsch (siehe Abschnitt 5). Anschließend werden die Befunde der lexikografischen Recherche mit Korpusdaten aus der erwähnten Doktorarbeit exemplarisch verglichen. Dabei wird die Relevanz der Beschreibung syntagmatischer und argumentstruktureller Eigenschaften von Neologismen in dem lexikografischen Informationsangebot aufgezeigt (siehe Abschnitt 6). Zuletzt wird für die Wichtigkeit mehrsprachiger Neologismenlexikografie plädiert, die sich insbesondere auf die freie Produktion und Übersetzung ins Deutsche als Fremdsprache ausgerichtet (siehe Abschnitt 7).

\section{Vorgehensweise und Untersuchungsparameter}

Im Zentrum der Analyse steht die fremdsprachige Produktionssituation (siehe 
Abschnitt 1). Diese Wörterbuchbenutzungssituation (vgl. Wiegand et al. 2010: 84) ist Ausgangspunkt und Bezugsrahmen der Untersuchung.

Für die Untersuchung einsprachiger Ressourcen wird angenommen, dass ein Sprecher des Spanischen als L1 ein neues Wort des Deutschen in einer konkreten kommunikativen Situation verwenden will. Der Benutzer ${ }^{1}$ weiß - oder zumindest vermutet - dass dieses Wort existiert. Er benötigt aber weitere Informationen zu seinem Gebrauch. Deswegen sucht er Hilfe in einem einsprachigen Nachschlagewerk. Er will eventuell semantische Informationen zu Distributionsbeschränkungen bekommen. Vor allem erwartet er Informationsangaben zu dem syntagmatischen Kombinationspotenzial und Gebrauchskontext $\mathrm{zu}$ finden, die ihm bei der adäquaten Verwendung des Wortes in der Fremdsprache helfen. Nun ergibt sich die Frage, ob er die gewünschten Informationen in den vorliegenden einsprachigen Wörterbüchern findet.

Für die Untersuchung zweisprachiger Wörterbücher wird ebenfalls die bereits geschilderte Benutzungssituation in Betracht gezogen. Dazu wird eine weitere Benutzungssituation berücksichtig, bei der angenommen wird, dass ein Sprecher des Spanischen als L1 das Konzept MEDIALE KOMMUNIKATION im Deutschen ausdrücken will. Ziel dieses Benutzers ist die freie Produktion in der Fremdsprache bzw. die umgekehrte Übersetzung. Die Suchtstrategie, die der Benutzer normalerweise verwendet, beruht auf muttersprachlicher Introspektion: Er kennt die lexikalischen Einheiten, die in seiner Muttersprache das gegebene Konzept versprachlichen und fragt sich, wie bzw. ob man auch „so etwas" auf Deutsch als L2 sagen kann. Dementsprechend sucht er Hilfe in einem bilingualen Wörterbuch. Er schlägt nach den verbalen Lexemen oder den Kernwörtern von komplexen Einheiten nach, die das Konzept in seiner Muttersprache lexikalisieren. Er will Entsprechungen suchen und finden und erwartet u.a. Informationsangaben $\mathrm{zu}$ dem syntagmatischen Kombinationspotenzial der Ziellemmata, die ihm dabei helfen, zunächst die passende Entsprechung auszuwählen und danach diese in der Fremdsprache adäquat zu verwenden. Nun ergibt sich hier ebenfalls die Frage, ob er die gewünschten Informationen findet.

Da die syntagmatischen Informationen in Wörterbüchern üblicherweise in Satzmuster und Beispielsätze einbezogen (bspw. Jemand chattet mit Jemandem oder Peter chattet mit seiner Familie) oder als morphosyntaktische Hinweise (Transitivität, Präpositionen, Kasusangaben, etc.) und Kontextangaben (zur Verdeutlichung des semantisch-syntaktischen Umfeldes) angeboten werden (vgl. Herbst 2009, Model 2010, Meliss 2015a, 2016, Fuentes Morán 2018), werden folgende Untersuchungsparameter in der Analyse besonders berücksichtigt: (i) Satzbauplan (SBP), (ii) Anzahl der Argumente und (iii) Valenz in syntaktischer sowie semantischer Hinsicht. Zudem wird das Interesse der Analyse zweisprachiger Wörterbücher auch auf (a) den Such-Auffindungsprozess und (b) die Disambiguierung der Ziellemmata gelegt. 


\section{Bestandsaufnahme lexikografischer Ressourcen zu Neologismen im Deutschen}

Unter den lexikografischen Ressourcen zu Neologismen muss man zwischen einsprachigen und zweisprachigen Angeboten unterscheiden.

Das einsprachige Angebot an lexikografischen Ressourcen zu Neologismen für das Deutsche basiert grundsätzlich auf Korpusarbeit (vgl. Engelberg und Lemnitzer 2009: 238-243) und ist namentlich in universitären und sprachwissenschaftlichen Forschungseinrichtungen zu finden. Das bevorzugte Medium ist das Internet. Dort zeigen Neologismendatenbanken eine stärkere Präsenz gegenüber (herkömmlichen) Online-Wörterbüchern. Diese Beobachtung schließt sich an die aktuelle Diskussion zu der verschwommenen Grenze zwischen Datenbanken und Wörterbüchern in der Online-Lexikografie an (vgl. Lew 2011: 246-248, Samaniego Fernández und Pérez Cabello de Alba 2011: 306-309). Die hier untersuchte Auswahl berücksichtigt diejenigen Online-Ressourcen, die bei der Abfrage in Google nach "Neologismenwörterbücher online" auf der ersten Google-Seite erscheinen, sowie diejenigen Neologismenwörterbücher in PrintFormat, die nicht älter als ca. 10 Jahre sind und für gewöhnlich in deutschen Universitätsbibliotheken zur Verfügung stehen². Die untersuchten Ressourcen sind das Neologismenwörterbuch des IDS-Mannheim sowie seine zweibändige Printversion Neuer Wortschatz. Neologismen im Deutschen 2001-2010 (vgl. Steffens und Al-Waldi 2015), die Neologismensammlung Die Wortwarte von Lemnitzer aus der Berlin-Brandenburgischen Akademie der Wissenschaften und das Deutsche Neologismenwörterbuch von Quasthoff (2007). Sie werden im Teilabschnitt 4 genauer vorgestellt und untersucht.

Bilinguale Neologismenwörterbücher für das Deutsche werden in der Regel im Rahmen eines umfangreichen lexikografischen Forschungsprojekts erstellt. Das IDS-Mannheim hat - zum Beispiel - die Materialien aus dem Projekt Neuer Wortschatz für das Sprachenpaar Deutsch-Russisch erweitert. Das Deutsch-russische Neologismenwörterbuch von Steffens und Nikitina (2016) - das auch online über das Plattform OWIDplus konsultierbar ist, richtet sich primär an russischsprachige DaF-Lernende, die eine Bedeutungserklärung suchen und ihren Wortschatz erweitern möchten. Weitere zweisprachige Neologismenwörterbücher im deutschen Kontext sind das Deutsch-chinesisches Neologismenwörterbuch von Dou (2004) und das Polnisch-deutsches Wörterbuch der Neologismen von Worbs, Markowski und Meger (2007). Das erste ist für die Rezeption (vgl. Engelberg und Lemnitzer 2009: 118-123) und den Wortschatzaufbau im Deutschen von chinesischsprachigen DaF-Lernenden konzipiert, während die Zielgruppe des zweiten Muttersprachler des Deutschen sind, die Polnisch lernen bzw. studieren. Konkret für das betreffende Sprachenpaar SpanischDeutsch liegt das Compendio temático de neologismos Alemán - Español: deutsche Neubildungen von Gierden Vega et al. (2010) vor. Es handelt sich um ein Kompendium neuer Wörter des Deutschen, deren Bedeutungserklärung auf Spanisch geschrieben ist. Es werden ggf. die spanischen Äquivalente sowie 
Gebrauchsbeispiele angeboten. Die stark ausgeprägte didaktische Funktion des Werks erkennt man an der thematischen Anordnung des Wortschatzes sowie an dem ergänzenden theoretischen Teil zur Wortbildung im Deutschen.

Sämtliche, hier dargestellten, bilingualen Wörterbücher - mit Ausnahme des Polnisch-deutschen Wörterbuchs der Neologismen - zielen auf die Erweiterung sozio-kultureller und historisch-politischer Kenntnisse der DaF-Lernenden durch den Wortschatzaufbau ab und haben somit nur diese spezifische Adressatengruppe im Visier. Daraus folgt, dass alle gerade erwähnten Wörterbücher primär auf folgende lexikografische Funktionen (vgl. Tarp 2013b: 465) ausgerichtet sind: kommunikative Funktionen bezüglich fremdsprachiger Rezeption und vor allem kognitive Funktionen (vgl. Tarp 2008: 44-54 bezüglich kommunikativer und kognitiver Situationen und Tarp 2008: 81-88 zu den entsprechenden Funktionen). Das heißt, dass sie dafür konzipiert sind, die Bedürfnisse eines Fremdsprache-Lernenden beim Textverstehen (vgl. Tarp 2008: 147149) und bei Erwerb bzw. Erweiterung seiner Kenntnisse in einem bestimmten thematischen Bereich (vgl. Tarp 2008: 163-166) zu befriedigen. Deshalb fallen diese Wörterbücher aus der vorliegenden Untersuchung heraus ${ }^{3}$.

\section{Informationsangebot in den aktuellen Neologismenwörterbüchern und Datenbanken für das Deutsche}

In Anbetracht der im Abschnitt 2 dargestellten Benutzersituation und der angeführten Parameter sind folgende einsprachige lexikografische Ressourcen zu Neologismen im Deutschen untersucht worden. Für jede Ressource folgen eine Beschreibung und ein Kommentar zu ihrer Adäquatheit für die betreffende Benutzersituation.

\subsection{Das Neologismenwörterbuch}

Das Neologismenwörterbuch ${ }^{4}$ ist Bestandteil des wissenschaftlichen lexikografischen Portals OWID des IDS-Mannheim (vgl. Müller-Spitzer: 2010). Es erfasst neue Wörter, die seit den 90er Jahren in das Deutsche eingegangen sind. Als empirische Grundlage dient das virtuelle Projektkorpus "neo", das vorwiegend Pressetexte aus DeReKo enthält, sowie eine selbst erstellte Wortkartei exzerpierter Print-, Hör- und Internetbelege. Dies wird durch wissenschaftliche Sekundärliteratur angereichert. Die Stichwortauswahl wird nach dreierlei Selektionskriterien getroffen. Die Wort-Kandidaten müssen (i) für den erfassten Zeitraum neu sein, (ii) der Allgemeinsprache angehören und (iii) dem deutschen Sprachgebrauch entsprechen.

Das Wörterbuchnetz OWID (vgl. Engelberg und Müller-Spitzer 2013: 1030-1033) bietet neben der Suchfeld-Option eine vielfältige Auswahl an Suchfunktionen an. Die Suche kann thematisch nach Fach- bzw. Sachgebieten oder chronologisch nach Dekaden eingeschränkt werden. Ebenfalls kann man durch 
die verschiedenen alphabetischen Register Zugang zu dem gesuchten Wort bekommen. Folgende Listen werden angeboten: Stichwort, Phraseologismen, Strichlemmata und verdeckte neue Wörter (sie sind keine Stichwörter. Sie sind in den Wortartikeln enthalten). Zudem besteht die Möglichkeit, eine erweiterte Suche zu tätigen. Hierbei kann die Suche durch folgende Filter verfeinert werden: Neologismentyp, Erscheinungsjahrzehnte, Wortart und Wortartmerkmale, Wortbildung und Wortbildungsproduktivität. Die Suche nach bestimmten Teilen bzw. Komponenten des Wortes ist gleichfalls möglich mithilfe der Kommandos ,"beginnt mit", ,"enthält" und „endet auf"5.

In der aktuellen online Fassung des Neologismenwörterbuchs (Stand: März 2018) sind alle in der oben erwähnten Doktorarbeit untersuchten, neuen MKV des Deutschen inventarisiert (siehe Abb. 1). Am Beispiel des Verbs simsen wird die Struktur eines Artikels beschrieben. Der Artikel besteht aus vier verschiedenen Informationsblöcken: (a) allgemeine Informationen (siehe Abb. 2), (b) Bedeutung und Verwendung (siehe Abb. 3), (c) Grammatik (siehe Abb. 4) und (d) weitere Informationen (siehe Abb. 5). Diese modulare Darstellung der lexikografischen Daten knüpft an den in dem lexikografischen Institut der Universität Aarhus geprägten Begriff monofunctional dictionary an (vgl. Bergenholtz 2012: 260). Sie vertreten die Auffassung, dass ein gutes Wörterbuch eine einfache Auffindung und einen schnellen Zugriff auf die gesuchten Daten ermöglichen soll. Dies geschieht, wenn die Daten auf die entsprechenden Benutzerbedürfnisse in einer bestimmten Situation ausgerichtet sind und folglich eine Datenüberflutung vermieden wird (vgl. Bergenholtz 2011). Die im Rahmen der Functional Theory gezeigte lexikografische Praxis tendiert zur Erstellung von mehreren verschiedenen funktionsgezielten Wörterbüchern auf der Grundlage einer gemeinsamen Datenbank (vgl. Bergenholtz 2012). Beispiele davon sind die Danish Music Dictionaries (vgl. Bergenholtz und Bergenholtz 2011). In derselben Linie entwickelt sich auch die lexikografische Lernplattform Base Lexicale $d u$ Français (vgl. Verlinde 2011). Poly- oder multifunktionale Wörterbücher müssen dann so transparent und flexibel bei der Darstellung ihrer Daten sein, dass man je nach Situation das passende monofunktionelle Wörterbuch daraus machen kann bzw. die benötigten Daten auswählen kann (vgl. Tarp 2009: 48-61). Bei elektronischen Wörterbüchern heißt das, dass der Benutzer vordefinieren kann, welche lexikografische Daten er (nicht) erhalten soll (vgl. Begriff „Benutzerschnittstelle" in Wiegand et al. 2010: 679), so wie es bei dem Neologismenwörterbuch der Fall ist. Ferner ist das gesamte Portal [OWID] hinsichtlich seiner Benutzerfreundlichkeit (vgl. Wiegand et al. 2010: 677) und Effizienz ( Usability) positiv bewertet (vgl. Heid 2011). Im Folgenden wird jeder Block detailliert dargestellt.

(a) Allgemeine Informationen: Schwerpunkt dieses Blocks sind die Informationen zur Entstehung, Frequenz und Morphologie des Wortes, wie in Abbildung 2 zu sehen ist. Unter dem Stichwort findet man Angaben zu der Lesart und zu der Dekade, in der das Wort aufgekommen ist, sowie ein 
Häufigkeitsdiagramm, das die zeitliche Verteilung der Gebrauchshäufigkeiten zu simsen ab 1990 darstellt. Danach folgen Informationen zu dem Neologismentyp, der Schreibung samt vorhandenen alternativen Schreibweisen und der Wortbildung.

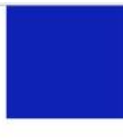

\section{simsen}

Lesart: 'schreiben und senden'

Neologismus der 90er Jahre

Benutzerhinweise

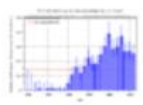

Neologismentyp:

\section{Neulexem}

Formvariante:

smsen

SMSen

Ausspracheangabe zu den

Formvarianten: [cs|cm'csən]

\section{Schreibung}

Worttrennung: $\quad \operatorname{sim} \mid$ sen

\section{Wortbildung}

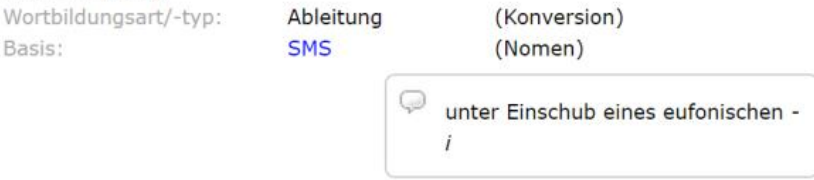

Abb. 2: Bildschirmausdruck des Eintrags zu simsen im Neologismenwörterbuch [22.02.18]

(b) Bedeutung und Verwendung: Dieser Block enthält eine kurze Definition, eine Sammlung typischer Verwendungen und eine Liste sinnverwandter Ausdrücke (siehe Abb. 3). Besonders innovativ ist das Angebot der als "typischer Verwendungen" etikettierten syntagmatischen Verbindungen, die eine hohe Frequenz nachweisen, ohne notwendigerweise idiomatisch zu sein. Sie veranschaulichen den typischen Gebrauch eines Wortes im syntagmatischen Zusammenhang und liefern vielfältige Informationen zu den lexikogrammatischen Eigenschaften des Wortes. Beispielsweise lässt sich aus den typischen Verwendungen von simsen erschließen, dass es oft durch Adverbien modifiziert wird (siehe ununterbrochen simsen, leidenschaftlich gern simsen), dass es häufig mit einem satzförmigen Direktobjekt auftritt (siehe simsen, wann die Versammlung stattfindet) und dass es üblicherweise in einem informellen bzw. familiären 
Kontext vorkommt (siehe dem Freund die Neuigkeiten simsen, mit der Freundin simsen).

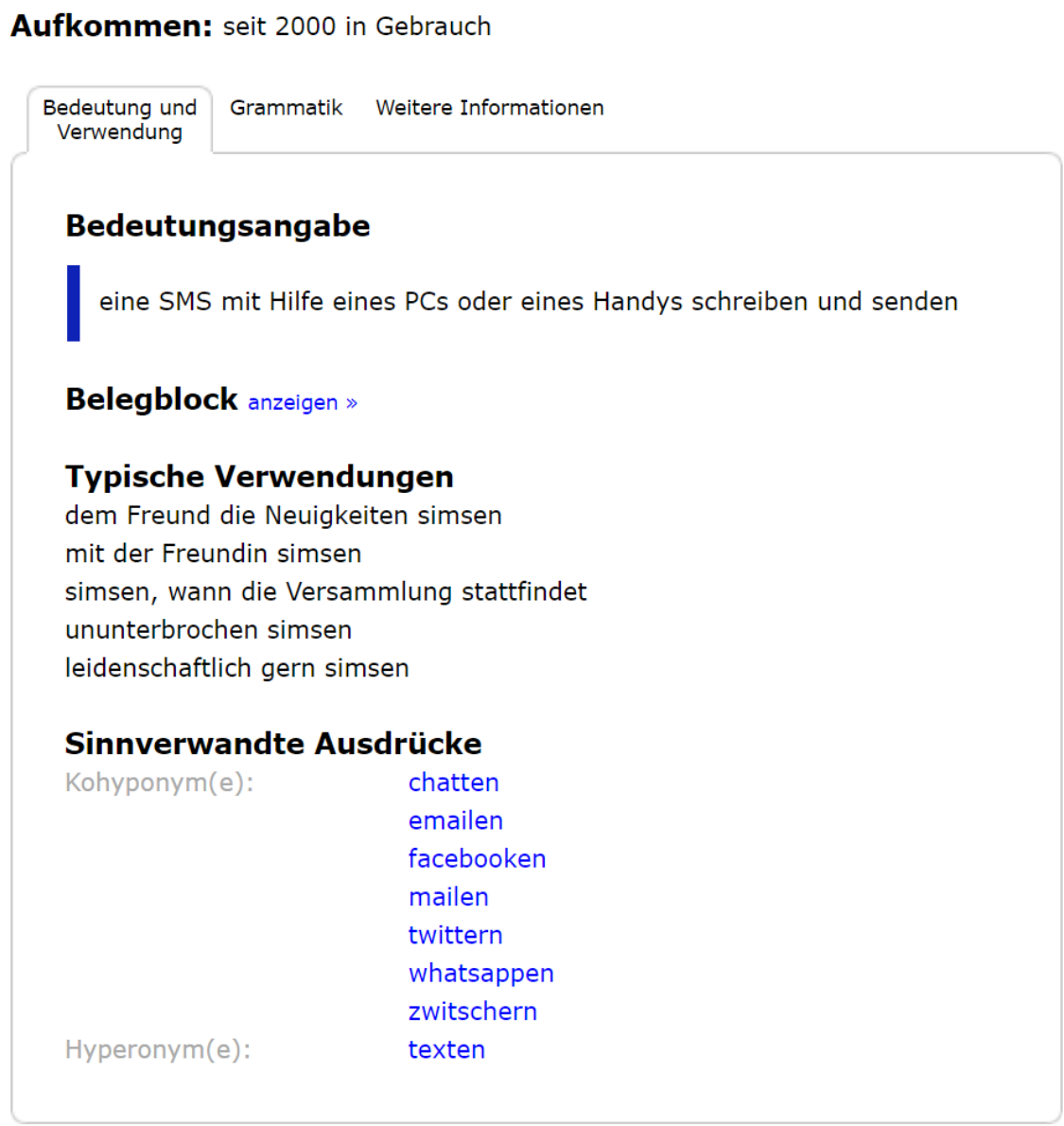

Abb. 3: Bildschirmausdruck des Eintrags zu simsen im Neologismenwörterbuch [22.02.18]

(c) Grammatik: Dieser Block bietet Informationen zu Wortart, Konjugation, Syntax und Wortbildungsproduktivität an (siehe Abb. 4). Die angebotene Beschreibung der syntaktischen Umgebung durch Phrasenmusterangaben informiert den Benutzer über die Eigenschaften obligatorischer und fakultativer Argumente des gesuchten Wortes, hier simsen. Sie zeigt - in abstrakter Form - das Valenzpotential des Verbs simsen, das aus den konkreten Realisierungen der Angabe "typische Verwendungen“ nur indirekt zu entnehmen ist (siehe Abb. 3). Beide komplementären Informationsangaben sind unerlässlich für die erfolgreiche Textproduktion in der Fremdsprache. 
Aufkommen: seit 2000 in Gebrauch

\begin{tabular}{|c|c|c|}
\hline $\begin{array}{l}\text { Bedeutung und } \\
\text { Verwendung }\end{array}$ & Grammatik & Weitere Informationen \\
\hline \multicolumn{3}{|c|}{ Grammatische Angaben } \\
\hline \multirow{2}{*}{\multicolumn{2}{|c|}{$\begin{array}{l}\text { Wortart: } \\
\text { Koniugation }\end{array}$}} & Verb (schwach) \\
\hline & & \\
\hline & simste \\
\hline \multicolumn{2}{|c|}{ Partizip Perfekt: } & gesimst \\
\hline \multicolumn{2}{|c|}{ Perfektbildung: } & mit haben \\
\hline \multicolumn{2}{|c|}{ Passiv: } & bildbar \\
\hline & & $\begin{array}{l}\text { meist im substantivierten Infinitiv } \\
\text { belegt; } \\
\text { finite Verbformen erst nach } 2000\end{array}$ \\
\hline \multirow{5}{*}{\multicolumn{2}{|c|}{ Syntaktische Umgebung: }} & jemand simst (jemandem) (etwas) \\
\hline & & jemand simst (mit jemandem) \\
\hline & & jemand simst, dass ... \\
\hline & & jemand simst, ob ... \\
\hline & & jemand simst, wer/wie/was/... ... \\
\hline \multicolumn{3}{|c|}{ Wortbildungsproduktivität } \\
\hline \multicolumn{2}{|c|}{ Präverbfügung: } & ansimsen, zurücksimsen \\
\hline \multicolumn{3}{|c|}{ Präverbfügungen erst nach 2000} \\
\hline
\end{tabular}

Abb. 4: Bildschirmausdruck des Eintrags zu simsen im Neologismenwörterbuch [22.02.18]

(d) Weitere Informationen: In diesem Block findet man - normalerweise weitere Angaben zu den paradigmatischen Informationen des Stichwortes und zu seiner Präsenz in anderen Wörterbüchern (siehe Abb. 5). Diese Informationen sind ebenfalls von großer Relevanz für die Textproduktion. Sie machen den Benutzer auf die Existenz vielfältiger Ausdrucksmöglichkeiten für dasselbe Konzept, das das konsultierte Stichwort lexikalisiert, aufmerksam. Die Darstellung des Stichwortes im Zusammenhang mit bedeutungsverwandten und teiläquivalenten Ausdrücken hilft dem Benutzer sowohl beim Wortschatzaufbau (kognitive Situation) als auch bei der stilistischen Verfeinerung seines Textes in Situationen der Textproduktion. 
Aufkommen: seit 2000 in Gebrauch

Bedeutung und Grammatik Weitere Informationen
Verwendung
Sprachreflexives
Alternativ zu dem jüngeren Verb simsen werden solche typischen
Verwendungen mit SMS (in der Lesart 'Kurzmitteilung') gebraucht wie: eine
SMS schicken, eine SMS senden, eine SMS schreiben. Sie sind in den IDS-
Textkorpora (derzeit noch) häufiger belegt als simsen.
simsen gehörte 2001 zu den von der Gesellschaft für deutsche Sprache in
Wiesbaden benannten "Wörtern des Jahres".
Vgl. im Portal OWID im Wörterbuch "Kommunikationsverben" den
Wortartikel
simsen
Für Tätigkeiten, die sich auf Kommunikation im Internet beziehen, sind seit
den 90er Jahren folgende Bezeichnungen aufgekommen und im
Neologismenwörterbuch Stichwort geworden: anmailen, bloggen, chatten,
emailen, entfolgen, entfreunden, facebooken, gruscheln, leaken, liken,
mailen, posten, retweeten, simsen, skypen, texten, twittern, voipen,
whatsappen, wischen, youtuben, zwitschern.
- Durkommen in Wörterbüchern
- Fuden - Wörterbuch der New Economy (2001)

Abb. 5: Bildschirmausdruck des Eintrags zu simsen im Neologismenwörterbuch [22.02.18]

Zusammenfassend kann man festhalten, dass das Neologismenwörterbuch ein lexikografisches Werkzeug ist, das sich sowohl für Muttersprachler als auch für DaF- und DaZ-Lernende zur Rezeption und Produktion deutscher Texte sowie zur Kenntniserweiterung in bestimmten Bereichen eignet, da der Benutzer je nach Bedürfnis und Situation die benötigten Daten selegieren kann.

\subsection{Die Wortwarte}

Das Projekt Die Wortwarte sammelt seit 2000 neue Wörter, die in den Onlineausgaben einiger Zeitungen sowie in den Onlinemagazinen Perlentaucher und 
heisse.de erschienen sind. Der Auswahlprozess erfolgt in zwei Etappen: Erst werden die Neuwort-Kandidaten mithilfe der in den Projekten des Programmbereichs Korpuslinguistik des IDS-Mannheim angebotenen Werkzeugen maschinell selegiert. Als Referenz für die Vorauswahl dienen die Wortlisten aus DeReKo (DeReWo). Danach sortiert Lemnitzer von Hand alle Ausdrücke, die keine neugebildeten Wörter sind, aus und von den letzteren wählt er nur diejenigen aus, die man nicht in die Kategorie der Okkasionalismen einschließen kann (vgl. Einleitung und Hintergrund in www.wortwarte.de). Die Wortsuche kann entweder alphabetisch, thematisch oder chronologisch eingegrenzt werden. Über ein Suchfeld, in das man das gesuchte Wort eingeben kann, verfügt die Webseite nicht. Folgende Verben aus der Gruppe der neuen MKV sind in der Neologismensammlung enthalten (Stand: März 2018): bloggen, mailen, simsen und twittern. Bei der Suche nach den neuen MKV wurde das alphabetische Kriterium eingesetzt. Im Folgenden wird das Informationsangebot der Wortwarte anhand des Eintrags zum Verb mailen exemplarisch beschrieben (siehe Abb. 6).

mailen, Verb

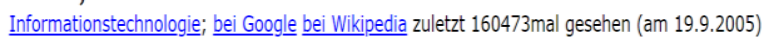

26 Oktober 200017 : 02 Hallo Gerald Meier, da es uns auch wirklich wichtig ist, dass Du uns wieder lieb hast, rödeln unsere Techniker seit ca einer Woche an Deinem Problem. Wenn Du näheres über den ganauen Stand der Dinge wissen willst, dann maile unseren Webmaster an : technik meinberlin . de ! Ich drücke die Daumen ! Tschüß - verregnete Grüsse Sabine vom meinberlin-Team Homepage : meinberlin . de 5 gerald meier - berlin Donnerstag 26 Oktober 2000 10:17 wann kümmert sich endlich jemand um den Zugang zum webkalender ! ?

http://www.meinberlin.de/tmh/gaestebuch/index.html

Abb. 6: $\quad$ Eintrag zu mailen in der Wortwarte [22.02.2018]

Wie in Abbildung 6 zu beobachten ist, besteht ein Eintrag in der Wortwarte aus folgenden Teilen:

- Stichwort („,mailen“)

- Angabe zur Wortart („Verb“). Bei Substantiven sind an dieser Stelle Informationen zu Genus sowie zu Genitiv- und Pluralbildung zu finden.

- Sachgebiet-Angabe (Informationstechnologie)

- Angabe zu Frequenzanzahl mit Datum („bei Google bei Wikipedia zuletzt 160473mal gesehen (am 19.09.2005)")

- Belegbeispiel mit Quelle zur Illustration des Wortes im Gebrauchskontext.

Die Wortwarte leistet eine empirisch fundierte Arbeit zur Sprachdokumentation, die ermöglicht, die Entwicklung des gegenwärtigen Wortschatzes des Deutschen zu beobachten. Die Neologismensammlung ist jedoch für Textproduktionszwecke wenig nützlich, da u.a. Angaben zu semantischer und syntagmatischer Kombinatorik fehlen. Außerdem kann die angebotene quantitative Information nur bedingt als Referenz genommen werden, da sie auf OnlineQuellen beruht und schnell veraltet. Sie hat eher einen impressionistischen Charakter. 


\subsection{Das Deutsche Neologismenwörterbuch}

Das Deutsche Neologismenwörterbuch von Quasthoff (2007) steht im Zusammenhang mit dem Projekt Deutscher Wortschatz der Abteilung für Automatische Sprachverarbeitung am Institut für Informatik der Universität Leipzig. Es erfasst ein Inventar von 2284 Stichwörtern, die sich durch ihre zunehmende Häufigkeitsschwelle und Mindestanzahl in den Korpora des Projekts im Zeitraum von 2000 bis Ende 2006 im Vergleich zu den Jahren 1995-1999 auszeichnen. Das umfangreiche Korpus besteht aus Zeitungstexten sowie Online-Ausgaben anderer Medien (vgl. Quasthoff 2007: 9). Das konsequent angewendete Häufigkeitskriterium führt zu einer Auffassung des Begriffs Neologismus im weitesten Sinne. Dementsprechend beinhaltet das Wörterbuch sowohl neu erschienene Wörter als auch etablierte Wörter, die vor Kurzem - und oft aus extralinguistischen Gründen - in der Alltagssprache auffällig frequent vorkommen (vgl. Quasthoff 2007: 5). Bezüglich der neuen MKV konnte kein Verb der Gruppe in dem Wörterbuch gefunden werden. De facto sind lediglich folgende drei Verben inventarisiert: abschaufeln, durchregieren und nachsteuern. Dennoch ist - im Zusammenhang mit den neuen MKV — das deverbale Substantiv Bloggen (neben den Substativen Blog, Blogger und Blogosphäre) im Wörterbuch registriert. Der Eintrag zu Bloggen wird hier benutzt, um die Eintragsstruktur exemplarisch zu beschreiben (siehe Abb. 7).

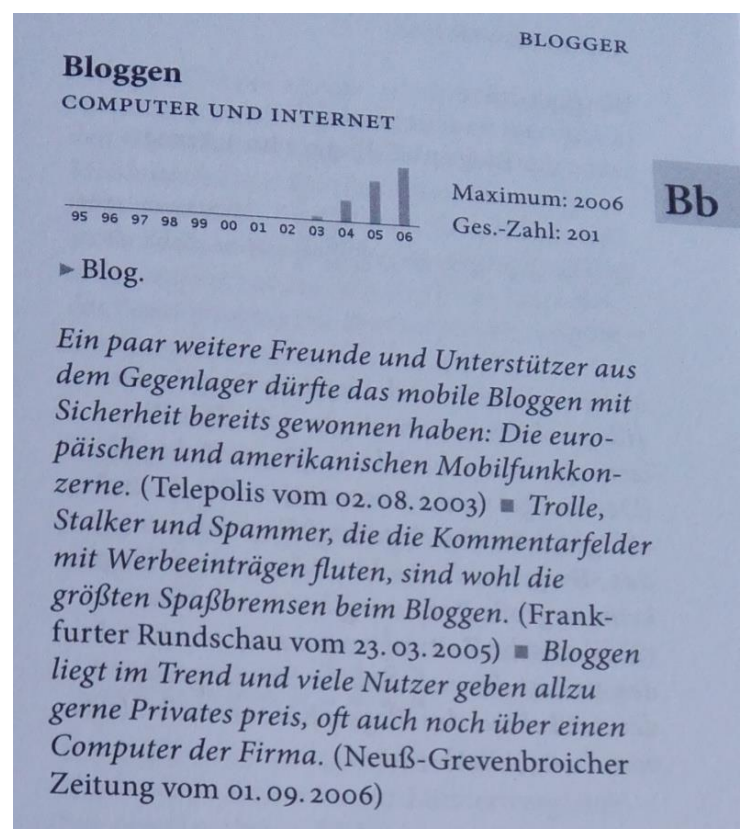

Abb. 7: Eintrag zu Bloggen in dem Deutschen Neologismenwörterbuch (Quasthoff 2007: 109) 
Der in Abbildung 7 illustrierte Eintrag zu Bloggen ist ein Verweiseintrag. Unter dem Stichwort als Überschrift findet man Information zum Sachgebiet (Angabe "Computer und Internet"), ein Häufigkeitsdiagramm und drei Belegbeispiele, die den Gebrauch des Wortes in den Medien veranschaulichen. Das Häufigkeitsdiagramm zeigt die Häufigkeit von Bloggen für jedes Jahr zwischen 1995 und 2006 im Vergleich. Überdies wird das Jahr der größten Häufigkeit (Angabe „Maximum: 2006") zusammen mit der Häufigkeit des Wortes im Gesamtzeitraum (Angabe "Ges.-Zahl: 201") hervorgehoben. Für weitere Informationen wird auf den Eintrag zu Blog verwiesen, in dem ein Erläuterungstext zu den Ursachen des auffälligen Wortgebrauchs zu finden ist. Haupteinträge können zudem Informationen zu alternativen Schreibweisen sowie eine kurze Definition enthalten. Dennoch müssen nicht alle Angaben immer vorhanden sein (vgl. Quasthoff 2007: 7).

Das Deutsche Neologismenwörterbuch von Quasthoff (2007) dient zum Erwerb und zur Erweiterung des enzyklopädischen Wissens und leistet eine sogfältige, lexikografische Dokumentationsarbeit, indem es den Zeitgeist der späten 90er und früheren Nullerjahre durch die Entwicklung des Wortschatzes wiederspiegelt. Als Hilfsmittel für die Textproduktion im Deutschen - zumal als Fremdsprache - wurde das Werk jedoch nicht konzipiert.

\section{Neologismen in bilingualen Wörterbüchern für das Sprachenpaar Spanisch-Deutsch}

Das Angebot an bilingualen allgemeinsprachlichen Wörterbüchern für das Sprachenpaar Spanisch-Deutsch ist relativ umfangreich (vgl. Domínguez Vázquez 2013, Meliss 2016). Die vorliegende Untersuchung beschränkt sich auf das online Angebot, da verschiedene lexikografische Studien und Umfragen zur Wörterbuchnutzung zeigen, dass sich das Internet als Medium in den letzten Jahren durchgesetzt hat (vgl. Domínguez Vázquez et al. 2013: 145-146, Meliss 2015b: 405-406, Fernández Méndez et al. 2016: 80 sowie Scheller-Boltz und Weinberger 2017: 10). Im Einzelnen werden folgende im Internet angebotene Wörterbücher der Analyse unterzogen: Die beiden Online-Wörterbücher Spanisch $\leftarrow \rightarrow$ Deutsch aus den lexikografischen Portalen von PONS und LEO und die Online-Version des Printwörterbuches Langenscheidt Spanisch. Die Auswahl lässt sich folgendermaßen begründen. Sowohl die oben zitierten Benutzerumfragen im Spanisch-Deutschen Kontext als auch andere lexikografische Studien europäischer Sprachen im Vergleich bestätigen, dass die Benutzer bevorzugt und am Häufigsten - die Online-Wörterbücher von PONS und LEO zu Rate ziehen (vgl. Meliss 2015b: 412-413, Fernández Méndez et al. 2016: 80 und Scheller-Boltz und Weinberger 2017: 11). Ergänzend soll die Analyse der Online-Version von Langenscheidt dazu dienen, Wörterbücher mit unterschiedlicher Nutzerbeteiligung (vgl. Wiegand et al. 2010: 90-91, Abel und Meyer 2016) miteinander zu vergleichen. Konkret wird das Informationsangebot der kollaborativen Wörterbücher (vgl. Abel und Meyer 2016: 253-262) 
PONS und LEO mit dem von dem rein institutionellen Wörterbuch Langenscheidt, in denen der Benutzer lediglich indirekt beteiligt wird (vgl. Abel und Meyer 2016: 263-272), kontrastiert (vgl. Fuertes-Olivera 2009: 103-107, Lew 2011: 230-231).

Die Untersuchung erfolgt aus der Perspektive eines Benutzers, dessen Zweck die Textproduktion in der Fremdsprache ist. Daher steht das syntagmatische Informationsangebot im Mittelpunkt des Untersuchungsinteresses, wie im Abschnitt 2 erklärt wurde. Die Analyse soll anhand der neuen MKV des Deutschen erfolgen. Sämtliche Verben sind jedoch nicht in allen drei untersuchten Wörterbüchern zu finden. Ein Überblick über die MKV, die in den genannten Wörterbüchern inventarisiert sind, liefert Abbildung 8.

\begin{tabular}{|c|c|c|c|c|c|c|c|}
\hline \multicolumn{4}{|c|}{ Ausrichtung ES $\rightarrow$ DE } & \multicolumn{4}{|c|}{ Ausrichtung DE $\rightarrow$ ES } \\
\hline \multicolumn{3}{|c|}{ Wörterbücher } & \multicolumn{2}{|c|}{ Stichwort } & \multicolumn{3}{|c|}{ Wörterbücher } \\
\hline $\begin{array}{c}\text { de.pons } \\
\text {.com }\end{array}$ & leo.org & $\begin{array}{l}\text { Langen } \\
\text { scheidt } \\
\text {-Online }\end{array}$ & ES & $\mathrm{DE}$ & $\begin{array}{c}\text { de.pons } \\
\text {.com }\end{array}$ & leo.org & $\begin{array}{l}\text { Langen } \\
\text { scheidt } \\
\text {-Online }\end{array}$ \\
\hline $\mathrm{A}_{\mathrm{B}}$ & A & I & bloguear & bloggen & A & A & I \\
\hline 马 & 马 & 马 & chatear & chatten & ㅍ & B & 马 \\
\hline I & I & A & postear & posten & $\mathrm{A}_{\mathrm{B}}$ & I & A \\
\hline $\mathrm{B}_{\mathrm{B}}$ & ב & ב & tuitear & twittern & $\exists_{B}$ & 马 & $\mathrm{G}$ \\
\hline$\varnothing$ & $\varnothing$ & $\varnothing$ & facebookear & facebooken & $\varnothing$ & $\varnothing$ & $\varnothing$ \\
\hline $\mathrm{a} !$ & a! & 3! & wasapear & whatsappen & $\varnothing$ & $\varnothing$ & $\varnothing$ \\
\hline$\varnothing$ & $\varnothing$ & 马 & skypear & skypen & $\mathrm{A} !_{\mathrm{B}}$ & G! & 马 \\
\hline \multirow[t]{2}{*}{$\varnothing$} & $\varnothing$ & $\varnothing$ & mailear & mailen & $\mathrm{a} !_{\mathrm{B}}$ & a! & a! \\
\hline & & & & simsen & 3! & 3! & 3! \\
\hline
\end{tabular}

Abb. 8: Überblick über die MKV, die in PONS, LEO und LangenscheidtOnline inventarisiert sind 6

Die im Rechteck eingeschlossenen MKV scheinen sich gegenüber den restlichen MKV im Wortschatz etabliert zu haben, da sie in den drei Wörterbüchern aufgeführt werden (siehe Symbol " $\mathbb{B}^{\prime \prime}$ in Abb. 8). Einige Einträge verdankt man jedoch dem kollaborativen Benutzer und nicht der Wörterbuchredaktion, wie der tiefgestellte Buchstabe B in Abbildung 8 zeigt $^{7}$. Das Verb simsen gehört auch zu den konventionalisierten Neologismen. Es unterscheidet sich von den anderen Verben darin, dass sich sein potenzielles Äquivalent esemesear in dem spanischen Wortschatz nicht festgesetzt hat. Aus diesem Grund bleibt es aus der vorliegenden Studie ausgeschlossen, da es in keinem Wörterbuch des Spanischen zu finden ist. Die Verben skypen und mailen sind ebenfalls in dem deutschen Wortschatz integriert, denn sie besitzen einen Eintrag im Wörterbuch. Das spanische Verb mailear hingegen ist in keinem der untersuchten Wörterbücher registriert, während das Verb skypear nur im Wörterbuch Langenscheidt-Online zu finden ist. Das umgekehrte Szenario stellt sich bei 
dem Sprachenpaar whatsappen-whastappear bzw. wasapear ein. Die spanischen Benutzer haben die an das orthografische Sprachsystem des Spanischen angepasste Form wasapear anerkannt und sie in das Wörterbuch eingetragen. Im Gegensatz dazu haben die deutsche Entsprechung zu whatsappear und die Verben skypen und skypear noch keinen Platz in den betreffenden Wörterbüchern gefunden. Diejenigen MKV, die nur für eine Sprache, d.h. entweder für das Spanische oder das Deutsche, abrufbar sind, tragen die Kennzeichnung ,!" in Abbildung 8.

Die Befunde zu den obigen Einträgen werden im Teilabschnitt 5.1 ausführlich präsentiert. Im Teilabschnitt 5.2 werden die entsprechenden Schlussfolgenrungen gezogen.

\subsection{Informationsangebot zu Neologismen in bilingualen Wörterbüchern}

In Anbetracht der im Abschnitt 2 dargestellten Benutzersituation ist das Informationsangebot zu Neologismen in den drei erwähnten zweisprachigen Wörterbüchern für das Sprachenpaar Spanisch-Deutsch untersucht worden. Anhand ausgewählter Wörterbuchartikel wird die Nützlichkeit dieses Angebotes bei (a) der Produktion in der Fremdsprache, (b) dem Such-Auffindungsprozess und (c) der Disambiguierung der Ziellemmata gezeigt.

\subsubsection{Neologismen in Pons-Online-Portal}

Das über das PONS-Online-Portal verfügbare bilinguale Wörterbuch Spanisch $\leftarrow$ Deutsch ist - im Prinzip — auf die Produktions- und Rezeptionsbedürfnisse beider Benutzergruppen ausgerichtet. Es hat zwar das Printwörterbuch als Ausgangspunkt, ist aber als Online-Wörterbuch neu konzipiert worden (vgl. Wiegand et al. 2010: 78-82, Engelberg und Storrer 2016: 34-35). Es wird grundsätzlich redaktionell betreut, bietet jedoch dem Benutzer die Möglichkeit, sich direkt und indirekt zu beteiligen (vgl. Abel und Meyer 2016: 252-253). Insofern kann der Benutzer neue Einträge erstellen und über die gegebene bzw. fehlende Informationen im Forum diskutieren. Direkten Zugang zu anderen lexikografischen Lern- und Forschungsressourcen bekommt der Benutzer ebenfalls. Dies garantiert aber nicht, dass das gesuchte Wort auch in den anderen Ressourcen zu finden ist. De facto muss der Benutzer immer eine neue Konsultation durchführen, da die Verlinkung nicht direkt zum Stichwort sondern auf die Startseite des ausgewählten Nachschlagewerks führt. In der von Engelberg und Müller-Spitzer (2013: 1029-1033) vorgeschlagenen Portaltypologie befindet sich das PONS-Portal an der Schnittstelle zwischen Wörterbuchsuchmaschinen und -netzen, da es eine Vereinheitlichung des Layouts und der Zugriffstruktur aber eine sehr hohe Eigenständigkeit der beinhalteten Wörterbücher aufweist (vgl. Engelberg und Storrer 2016: 54). 
Im Folgenden werden die Einträge $\mathrm{zu}$ posten/postear (siehe Abb. 9), zu mailen (siehe Abb. 10), zu twittern/tuitear bzw. twittear (siehe Abb. 11) und zu wasapear [whatsappen] (siehe Abb. 12) aus dem PONS-Portal in Verbindung mit der Frage nach der Nützlichkeit des Informationsangebots zu den Schwerpunkten (a)-(c) exemplarisch analysiert.

Der Eintrag zu postear und der zu posten haben verschiedene Autorschaft. Der erste ist von der PONS-Redaktion verfasst worden, während der letzte von einem Benutzer erstellt worden ist, wie in Abbildung $9 \mathrm{zu}$ sehen ist. Dies lässt sich ebenfalls an dem unterschiedlichen Informationsangebot beider Artikel bemerken. Der Benutzer findet nur Hinweise zu der semantischen und syntaktischen Valenz des Verbpaares posten/postear, wenn er von dem Spanischen ausgeht (Suche nach postear = "postear algo" $\rightarrow$ "etw posten").

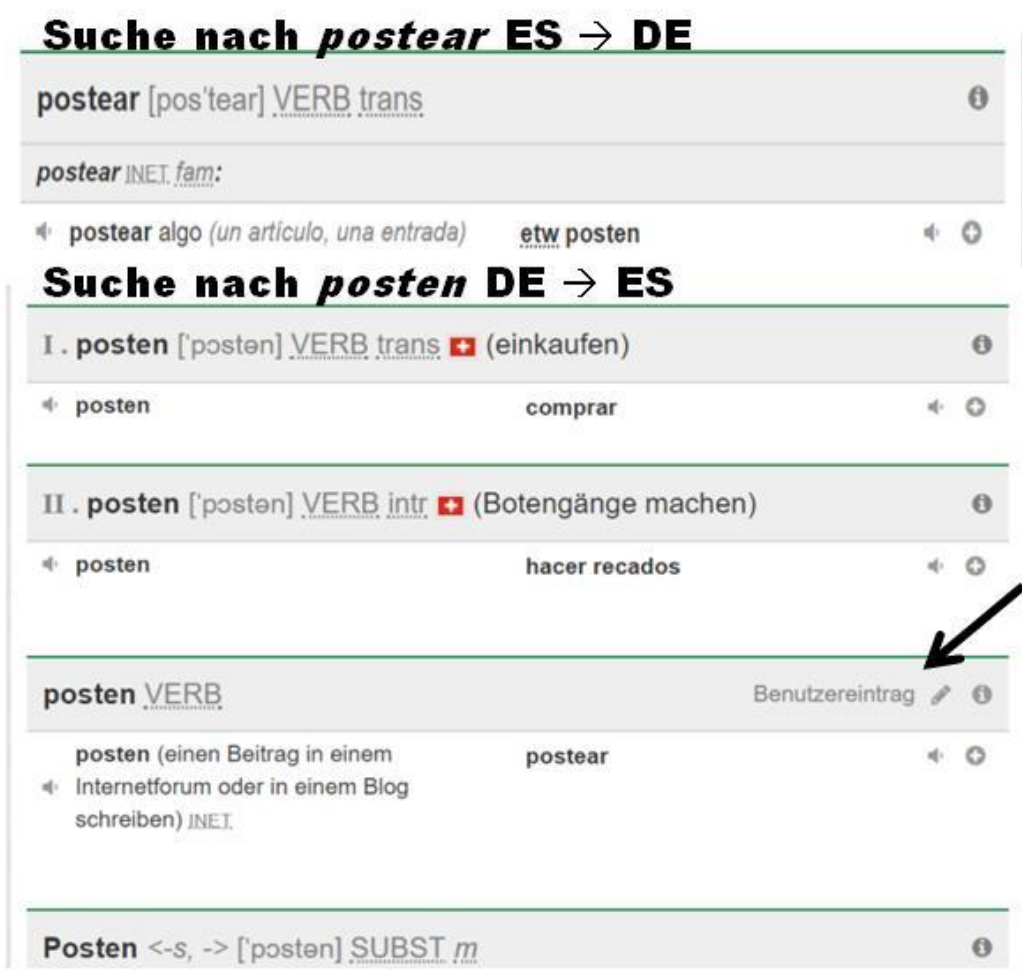

Abb. 9: Bearbeitete Bildschirmausdrucke der Einträge zu postear und posten in Pons-Online [06.03.18]

Für Rezeptionszwecke hingegen ist die Konsultation in beiden Ausrichtungen erfolgreich, da sowohl der ELE- als auch der DaF-Lernende den Ausdruck in seiner Muttersprache findet, der ihm hilft, die Bedeutung in der Fremdsprache zu deuten. Der Eintrag zu posten bietet zusätzlich eine ausführliche Bedeu- 
tungserklärung an. Bezüglich der Ausrichtung DE $\rightarrow$ ES lässt sich das Stichwort posten, das mehrdeutig ist, anhand von kategoriellen Informationen (bspw. "VERB" vs. "SUBST m") und Angaben zum Sachgebiet bzw. Kontextgebrauch (,einkaufen“, „Botengänge machen“ oder „INET“) gut disambiguieren.

Ein anderes Szenario stellt der Artikel zu mailen dar. Wie in Abbildung 10 zu sehen ist, findet der Benutzer zwei verschiedene Einträge zu mailen, die sich in der Information zu der AS voneinander unterscheiden. Beide führen zu sehr ähnlichen aber jedoch nicht identischen Entsprechungsvorschlägen im Spanischen. Wenn ein DaF-Lernender das Verb mailen in diesem Wörterbuch nachschlägt, um Informationen zu der adäquaten Verwendung dieses Verbes zu erhalten, muss er entscheiden können, welche von den angebotenen Varianten (d.h. die Dativ- oder die Präpositionalvariante von mailen) sich am besten für seinen Zweck eignet. Da keine Gebrauchsbeispiele angeboten werden, kann ihm diese Entscheidung schwer fallen. Wenn er sich für die Präpositionalvariante entscheiden sollte, muss er erst die Angaben "mailen (an) (akk)“ richtig interpretieren können. Er muss schon vorher wissen, dass sich die Angabe "(akk)" auf die Kasusrektion der in der Angabe "(an)“ erwähnten Präposition bezieht. Es muss sich dann um einen fortgeschrittenen DaF-Lernenden oder einen kundigen bzw. sehr erfahrenen Benutzer (vgl. Wiegand 1998: 505-508) handeln. Wenn aber diese Kenntnisse fehlen, muss der Benutzer nicht zwangsläufig zu dem richtigen Schluss kommen, dass das Verb mailen mit einem Präpositionalobjekt auftritt, dessen Präposition (an) Akkusativ regiert". Er kann gleichfalls auf die falsche Interpretation kommen, dass das Verb mailen ein Akkusativobjekt und ein Präpositionalobjekt fordert, bei dem der passende Kasus zu der Präposition an unbekannt ist". Über die semantische Valenz von mailen oder die mögliche lexikalische Besetzung der Argumente erhält der Benutzer an dieser Stelle ebenfalls keine Auskunft. Die syntagmatischen Informationen sind unvollständig. Das ist ein Beispiel von der Problematik hinsichtlich der Kodifizierung von Valenzangaben in Wörterbüchern, die schon Herbst (2009: 287-289) in Bezug zu Lernerwörterbüchern für das Englische angesprochen hat.

\begin{tabular}{|c|c|c|}
\hline mailen $V E R B$ & Benutz & i \\
\hline jdm mailen & enviar un e-mail (a alguien) & $\leftrightarrow \oplus$ \\
\hline mailen (an) (akk) & $\begin{array}{l}\text { enviar un sms, mail... (a } \\
\text { alguien) }\end{array}$ & $\Leftrightarrow \oplus$ \\
\hline
\end{tabular}

Abb. 10: Eintrag zu mailen in Pons-Online [06.03.18]

Darüber hinaus kann der Benutzer nur Zugriff zu dem Verb mailen erhalten, wenn er schon vorher das Verb kennt und gezielt nach ihm sucht. Ein spanischsprachiger Benutzer, der sowohl die Verben enviar [senden], mandar [schicken] und escribir [schreiben] als auch die nominalen Komponente der Konstruktio- 
nen correo [Post] und e-mail [E-Mail] nachschlägt, wird nicht unmittelbar zu mailen geführt. Erst nach weiteren Konsultationen und über das deutsche Substantiv Mail gelangt er an den Benutzereintrag zu mailen.

Die in Abbildung 11 abgebildeten Einträge zu tuitear und twittern bestätigen ebenfalls, dass die Äquivalenzrelationen bzw. das Äquivalenzangebot in PONS-Online unsystematisch sein kann. Beispielsweise kommt der Benutzer von twittern im Deutschen sowohl zu tuitear als auch zu twittear im Spanischen aber nicht umgekehrt. Die Schreibvariante twittear entspricht keinem spanischen Eintrag oder Stichwort im Wörterbuch. Die Suche nach tuitear führt zum Verb twittern ohne weitere morphosyntaktische Informationen (z.B. kein Hinweis auf Transitivität). Dieses unsystematische und unvollständige Informationsangebot hat möglicherweise seine Ursache in der Autorschaft der Beiträge, da sie von Benutzern und nicht vom Redaktionsteam erfasst worden sind. Daraus schließt sich, dass PONS als ein kollaborativ-institutionelles Wörterbuch verstanden werden soll, bei dem die Benutzerbeiträge - a priori - redaktionell unbearbeitet bleiben (vgl. Lew 2011: 237, Abel und Meyer 2016: 260-261).

\section{Suche nach tuitear ES $\rightarrow$ DE}

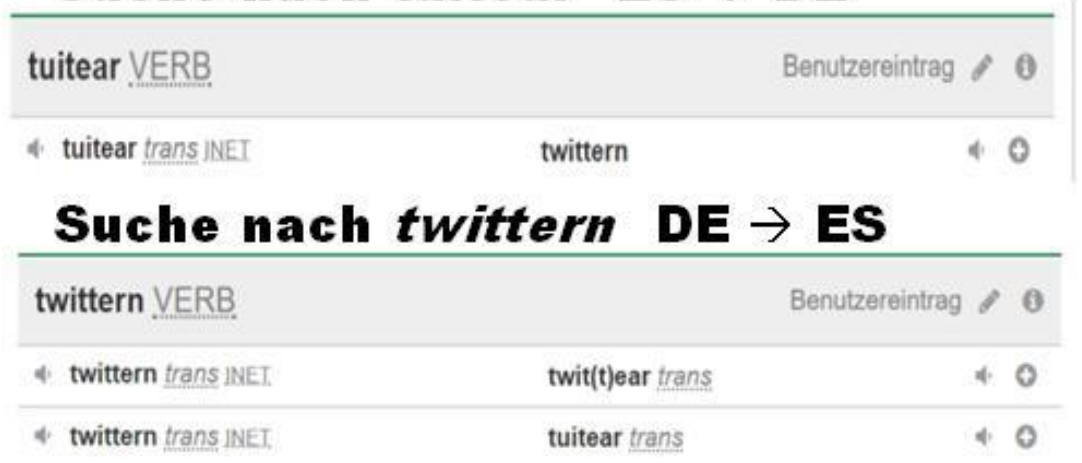

Abb. 11: Bearbeitete Bildschirmausdrucke der Einträge zu tuitear und twittern in Pons-Online [06.03.18]

Ähnliche Kritikpunkte kann man bezüglich des Eintrags zu wasapear [whatsappen] vorbringen, obwohl es sich nicht um einen Benutzereintrag handelt (siehe Abb. 12). Dem Benutzer wird eine Entsprechung zu dem nachgeschlagenen spanischen Stichwort wasapear geliefert. Das syntagmatische Informationsangebot - ohne ergänzende Gebrauchsbeispiele - erweist sich als unzureichend für die Produktionszwecke des DaF-Lernenden. Beispielsweise wird dem DaF-Lernenden die Information zu dem Kasus des Reflexivpronomens sich in dem Ausdruck sich über WhatsApp schreiben nicht geliefert. Die Angabe ist folglich für einen Benutzer mit geringem Sprachniveau mangelhaft. 


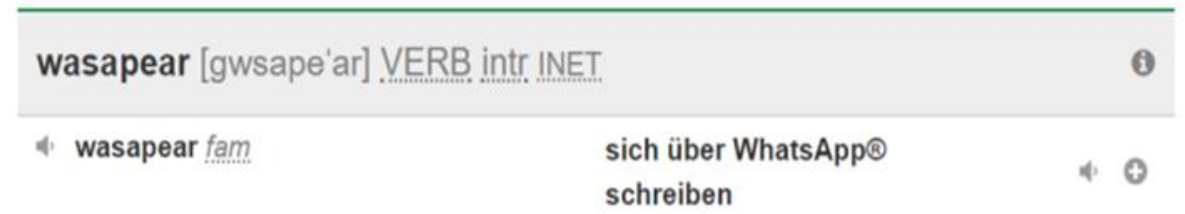

Abb. 12: Eintrag zu wasapear in Pons-Online [06.03.18]

\subsubsection{Neologismen in Leo-Portal}

Die Leo $\mathrm{GmbH}$ bietet vielfältige zweisprachige Wörterbücher in Verbindung mit dem Deutschen sowie einen Trainer- und Forumbereich für die jeweiligen Sprachen. Die lexikografische Ressourcen des LEO-Portals sind seit ihrem Ursprung als reines Onlineangebot mit kooperativem Charakter konzipiert (vgl. Engelberg und Storrer 2016: 39 und Abel und Meyer 2016: 283). Die Benutzereinträge werden von Sprachexperten geprüft. In diesem Sinne weisen sich die LEO-Wörterbücher als semi-kollaborativ aus (vgl. Abel und Meyer 2016: 261-263). Dazu gehört auch das bilinguale Wörterbuch Spanisch $\leftarrow \rightarrow$ Deutsch. Zusätzlich stellt LEO externe Verlinkungen zu anderen Wörterbüchern und didaktischen Sprachressourcen zur Verfügung, über die der Benutzer weitere Informationen zu dem gesuchten Ausdruck erhalten kann. Allerdings steht diese Verlinkung nicht für alle Stichwörter bereit, da es kein systematisches, redaktionell ausgearbeitetes Angebot ist, sondern automatisch generiert wird.

Im Folgenden werden die Einträge zu twittern/tuitear (siehe Abb. 13), zu posten/postear (siehe Abb. 14 und Abb. 15), zu mailen (vgl. Abb. 16) und zu wasapear [whatsappen] (siehe Abb. 17) aus LEO illustriert und die Nützlichkeit des entsprechenden Informationsangebots in Verbindung mit den Schwerpunkten (a)-(c) kommentiert.

Mit Unabhängigkeit von der Ausgangssprache wird der Benutzer in der Regel zu einem einzigen zweisprachigen Artikel geführt. Dies könnte damit zusammenhängen, dass LEO die typischen Eigenschaften eines virtuellen Wörterbuchsportals aufweist (vgl. Engelberg und Storrer 2016: 53). Sein Informationsangebot ist so vereinheitlicht, dass die Eigenständigkeit der integrierten Wörterbücher sehr schlecht zu erkennen ist. Beispielsweise kommen sowohl der Benutzer, der nach tuitear, als auch der Benutzer, der nach twittern nachgeschlagen hat, zu demselben Doppeleintrag zu tuitear/twitter, der in Abbildung 13 gezeigt wird. Das Wörterbuch LEO zeichnet sich u.a. dadurch aus, dass es dem Benutzer ausführliche morphosyntaktische Informationen anbietet. Zum Beispiel kann der Benutzer in dem Artikel zu tuitear/twittern den Info-Button anklicken und sich die ganze Konjugation des jeweiligen Verbs tabellarisch zeigen lassen, wie in Abbildung 13 illustriert wird. Diese Daten sind für die Textproduktion zwar hilfreich aber nicht ausreichend. Konkrete Angaben zur Valenz oder Argumentanzahl von twittern findet der Benutzer nämlich nicht. Der Hinweis ",- in sozialen Netzwerken" ist konfus. Diese 
Präpositionalphrase kann als typische Verwendung bzw. häufiger Kookkurrenzpartner des Verbs (in sozialen Netzwerken twittern) interpretiert werden. Es kann sich aber auch um eine Kontext- bzw. Sachgebiet-Angabe handeln. Weitere Gebrauchsbeispiele erhält man für dieses Verb nicht.

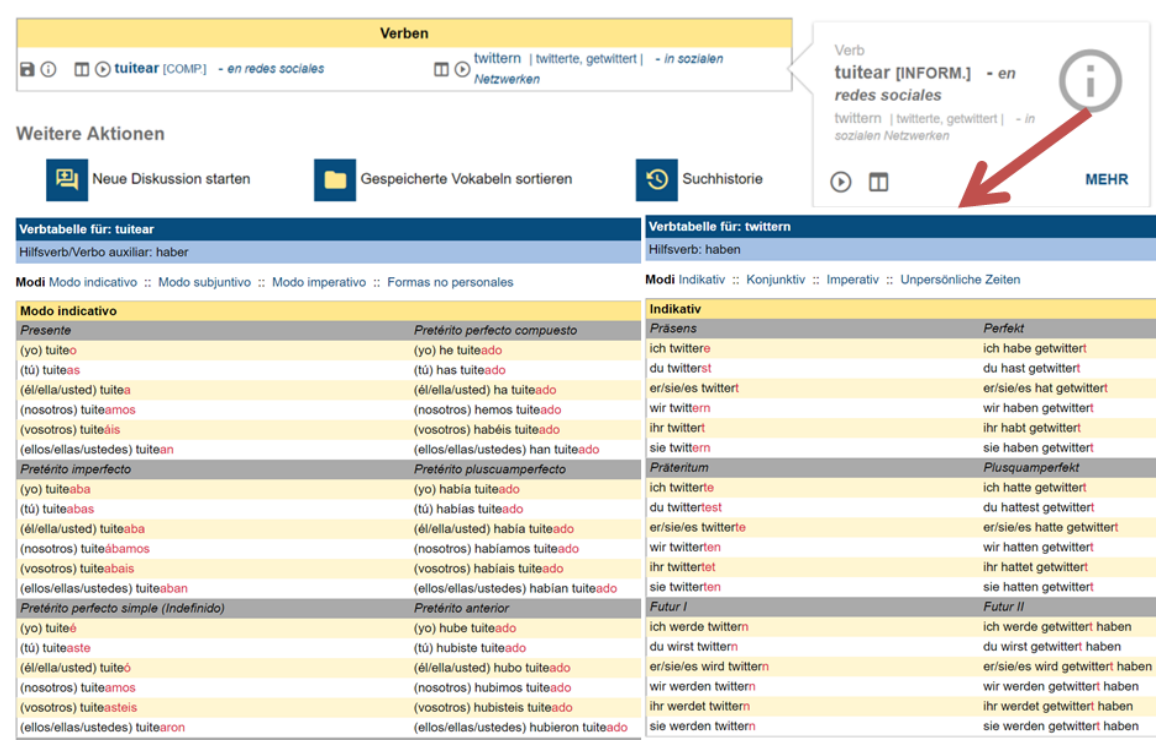

Abb. 13: Bearbeitete Bildschirmausdrucke der Einträge zu twittern und tuitear in leo.org [06.03.18]

Eine Ausnahme bilden die Einträge für das Verbpaar posten/postear, da die Suche nach dem jeweiligen Verb je nach Ausgangssprache $\mathrm{zu}$ unterschiedlichen Einträgen leitet, wie die Abbildungen 14 und 15 veranschaulichen. Der Grund dafür ist, dass sich das Stichwort posten im Deutschen auf zwei homophone Wörter beziehen kann: das Substantiv der Posten und das Verb posten, wie Abbildung 15 bezeugt. Die Suche nach postear hingegen führt direkt zu dem spanischen Eintrag "postear algo" mit seinem deutschen Pendant „etw.Akk posten" (siehe Abb. 14). Da findet der Benutzer semantische und syntaktische Valenzangaben, die er für die adäquate Verwendung des Verbs und seiner Argumente im Deutschen benötigt. Dazu steht eine kurze Bedeutungserklärung "- etwas in einem Weblog schreiben" zur Verfügung, die gleichzeitig als Kontexthinweis dient. Weitere morphosyntaktische Informationen kann der Benutzer über den Info-Button erhalten.

$\mathrm{Zu}$ diesen Informationen gelangt auch der Benutzer, der "posten" nachgeschlagen hat. Er bekommt sie allerdings auf eine andere Art und Weise dargestellt. Der Artikel zum deutschen Stichwort "posten" teilt das Informationsangebot in zwei Blöcke, wie man in Abbildung 15 sehen kann. Der erste trägt die Überschrift „Substantive“. Der zweite lautet „Verben“. Logischer- 
weise hätte der Benutzer denken können, dass er, wenn er einmal den „Substantive"-Block gemieden und sich direkt auf den "Verben"-Block gerichtet hat, dort die Informationen erhalten würde, die ausschließlich das Verb posten betreffen. Das ist jedoch nicht der Fall. Der Benutzer muss erst diejenigen verbalen Ausdrücke und Kollokationen aussortieren, die mit dem Verb posten nicht in Verbindung stehen. Dann bleiben drei passende Einträge üblich. Diese sind in Abbildung 15 eingerahmt worden. Die drei Einträge auf der deutschen Seite beinhalten fast identische Angaben. Sie korrelieren allerdings mit drei verschiedenen Einträgen auf der spanischen Seite. Der erste Entsprechungsvorschlag zu "etw. Akk posten“ ist "postear algo" [etwas posten], der zweite „publicar un post" [einen Post veröffentlichen] und der dritte "publicar algo" [etwas veröffentlichen]. Die drei Einträge werden durch weitere Angaben kontextuell eingegrenzt (z.B. „in sozialen Netzwerken“). Unbekannt bleibt noch der Grund, warum das vielfältige Angebot an Ausdrucksmöglichkeiten nur für das Spanische und nicht auch für das Deutsche vorhanden ist.

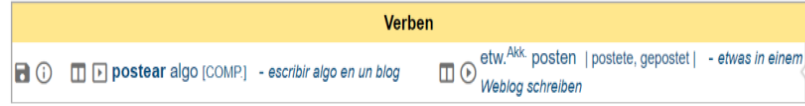

Weitere Aktionen

밀 Neue Diskussion starten postear algo [INFORM.] escribir algo en un blog

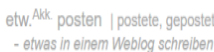

๑

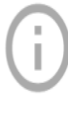

MEHR

Abb. 14: Eintrag zu postear in leo.org [06.03.18]

\begin{tabular}{|c|c|c|}
\hline \multicolumn{3}{|c|}{ Substantive } \\
\hline (a) (1) & (i) el apostadero & (1) (1) der Posten Pl: die Posten \\
\hline a (1) & (C) el puesto & (1) (1) der Posten Pl: die Posten \\
\hline a (1) & (1) el cargo [mg.] & (1) (-) der Posten Pl: die Posten \\
\hline (a) (1) & (1) la columna [KOMM ][MIRTSCH] & (1) ( ) der Posten Pl. die Posten \\
\hline (a) (1) & (1) la partida [Kомм] & (1) () der Posten Pl: die Posten \\
\hline (a) (1) & (1) el rubro [MRTSCH] & (1) (ㄱ) der Posten Pl: die Posten \\
\hline $\mathbf{a}(1)$ & 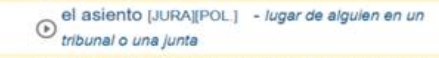 & $\varpi(\odot)$ der Posten Pl: die Posten - Dei Gericht oder in \\
\hline a (1) & [ las transacciones corrientes $t . P .[$ MIRTSCH.] & - die laufenden Posten $P$ l. \\
\hline $\mathbf{a}(1)$ & $\square$ balanza de operaciones corrientes [FINAN] & $\square$ die Bilanz der laufenden Posten \\
\hline \multicolumn{3}{|c|}{ Verben } \\
\hline $\mathbf{a}(1)$ & 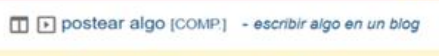 & 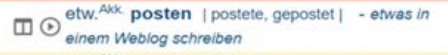 \\
\hline $\mathbf{a}(1)$ & $\square$ publicar un post [COMP] - blog & 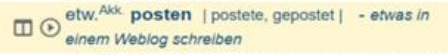 \\
\hline (a) (1) & (1) (1) publicar algo [Comp] - comentario, en redes soc. & $s \square(\odot)_{\text {Netzwerken }}^{\text {etw, Avk posten I postete, gepostet | - in sozialen }}$ \\
\hline $\mathbf{a}$ (i) & (1) renunciar a un cargo [ADMIN] & $\square$ von einem Posten zurücktreten \\
\hline $\mathbf{a}$ (1) & ๑๑ renunciar a su cargo & $\square$ seinen Posten verlassen \\
\hline $\mathbf{a}$ (1) & ocupar un puesto importante & [ einen wichtigen Posten innehaben \\
\hline $\mathbf{a}$ (1) & 巴๑ destituir a alguien de su cargo & 曰jmdn. von seinem Posten entheben \\
\hline
\end{tabular}

Abb. 15: Bearbeiteter Ausschnitt aus dem Eintrag zu posten in leo.org [06.03.18] 
Die Abbildungen 16 und 17 dienen zur Illustration der Einträge zu dem deutschen Verb mailen und dem spanischen Substantiv e-mail einerseits, und zu dem spanischen Verb wasapear und dem Substantiv WhatsApp anderseits. Sie bestätigen, dass die Auffindung komplexer Einheiten in LEO normalerweise erfolgreich ist und dass man auch über komplexe Einheiten Zugang zu einfachen Einheiten erhält. Beispielsweise führt die Suche nach dem spanischen Substantiv $e$-mail sowohl zu den komplexen Einheiten escribir un e-mail a alguien [jemandem eine E-Mail schreiben] und enviar algo a alguien por correo electrónico [jemandem etwas per elektronischer Post senden] im Spanischen und jemandem etwas per E-Mail senden im Deutschen als auch zum Verb mailen (siehe Abb. 16). Genauso gelangt man über das deutsche Stichwort WhatsApp zu dem deutschen Ausdruck per WhatsApp schreiben bzw. jemandem (Nachrichten) über WhatsApp schreiben und $\mathrm{zu}$ dem spanischen Ausdruck mandar wasaps a alguien [jemandem WhatsApp-Nachrichten schicken] aber auch zum Verb wasapear (siehe Abb. 17).

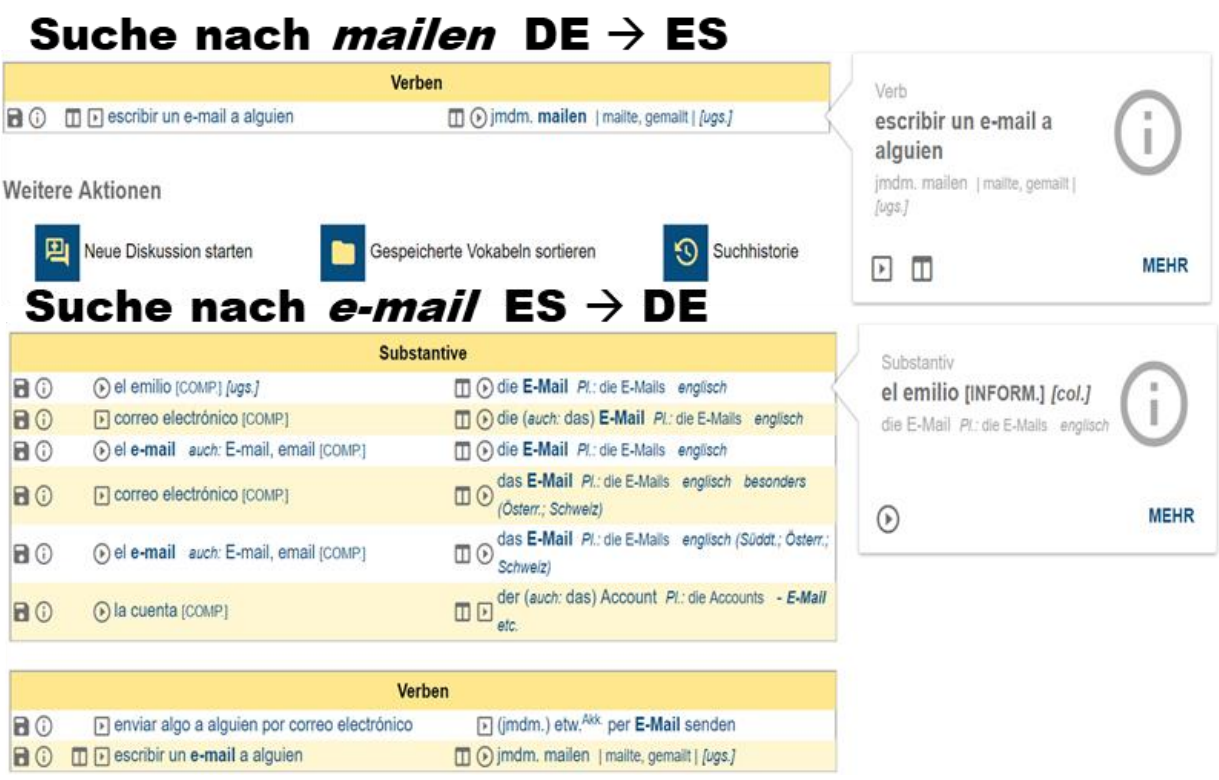

Abb. 16: Bearbeitete Bildschirmausdrucke der Einträge zu mailen und e-mail in leo.org [12.03.18] 


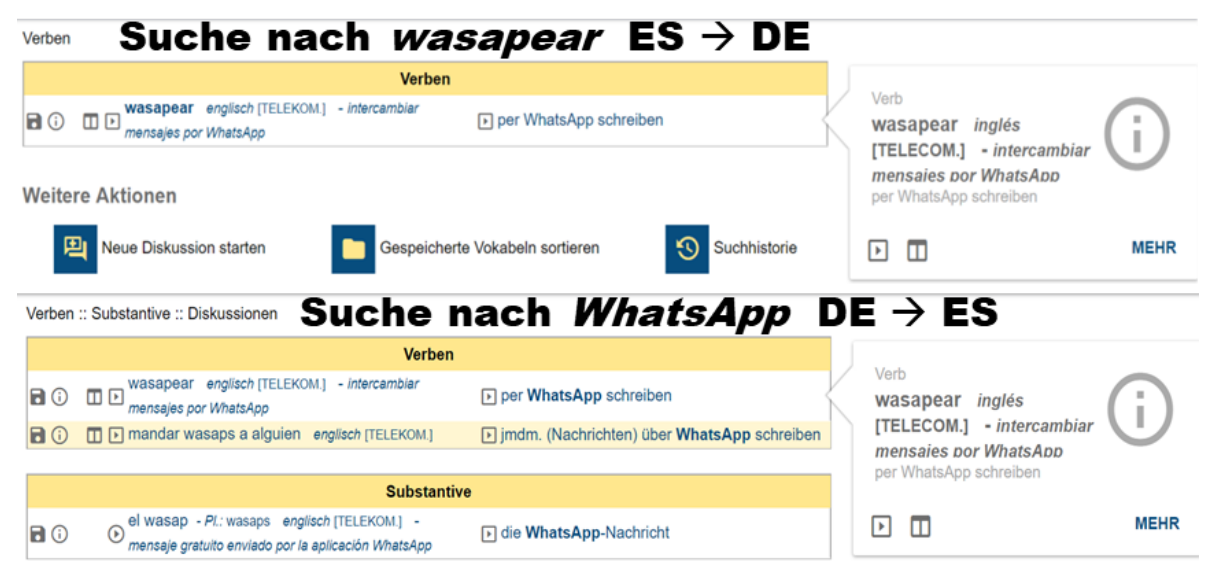

Abb. 17: Bearbeitete Bildschirmausdrucke der Einträge $\mathrm{zu}$ wasapear und WhatsApp in leo.org [12.03.18]

\subsubsection{Neologismen in Langenscheidt-Online}

Das im Internet angebotene zweisprachige Wörterbuch Spanisch $\leftrightarrow \rightarrow$ Deutsch von Langenscheidt fußt auf seinem gedruckten Pendant und soll eher als eine digitalisierte Version des Printwerks verstanden werden, die in dem neuen Portal des Langenscheidt Verlages integriert ist. Das Wörterbuchportal funktioniert wie eine Suchmaschine. Trotzdem zeigt es hinsichtlich Layout Ähnlichkeiten zu Wörterbuchnetzen auf (vgl. Engelberg und Storrer 2016: 52-53). Das Wörterbuch befindet sich noch in dem Medienwandel-Prozess (vgl. Wiegand et al. 2010: 88-89), da es das Potenzial des digitalen Formates nicht optimal ausgeschöpft hat (vgl. Debus-Gregor und Heid 2013: 1009-1011, Engelberg und Storrer 2016: 34-35). Beispielsweise verfügt es weder über externe Verlinkungen zu anderen lexikografischen Ressourcen noch über interne Verlinkungen zu ergänzenden Materialien oder zu Diskussionsforen. Der Benutzer hat dennoch die Möglichkeit, durch ein Feedback-Formular seine Meinung zu äußern.

Im Folgenden werden die Einträge zu twittern/tuitear (siehe Abb. 18), zu posten/postear (siehe Abb. 19), zum deutschen mailen und zum spanischen wasapear [whatsappen] (siehe Abb. 20) aus Langenscheidt in Verbindung mit der Frage nach der Nützlichkeit des Informationsangebots zu den Schwerpunkten (a)-(c) kommentiert.

Abbildung 18 präsentiert die Einträge zu tuitear/twittern aus Langenscheidt-Online im spanisch-deutschen Vergleich. Auf den ersten Blick wird schon auffällig, dass der deutsche Eintrag zu twittern ausführlicher als der spanische Eintrag zu tuitear ist. Er enthält über die Entsprechungsvorschläge in der Zielsprache (vgl. "usar Twitter" und "tuitear") und die Angaben zu Aussprache und Wortart des Stichwortes (vgl. „['tvitərm] v/i“) hinaus zusätzliche 
Gebrauchsbeispiele zum Stichwort aber auch zu den vorgeschlagenen Äquivalenten in der Zielsprache, eine kurze Bedeutungserklärung des Stichwortes (vgl. „ $\approx$ den Internetdienst Twitter nutzen") sowie Markierungen zu Sprachregister der vorgeschlagenen Äquivalenten in der Zielsprache (vgl. "UMG") und Sachgebiet des Stichwortes (vgl. „INTERNET"). Das heißt, dass der Benutzer, der zunächst nach dem spanischen Verb tuitear gesucht hat, noch eine weitere Konsultation tätigen muss, um an diese Daten zu kommen. Auffällig wird auch auf den zweiten Blick, dass die Angaben zu Transitivität bzw. Intransitivität im Widerspruch zu den syntagmatischen Informationen stehen, die aus den Beispielen hergeleitet werden können. Laut der Angabe zur Wortart, die neben dem Stichwort ",twittern" steht, ist das Verb intransitiv (vgl. „, $\left.\mathrm{v} / \mathrm{i}^{\prime \prime}\right)$. Im unteren Beispiel wird das Verb aber transitiv verwendet (vgl. „er hat das Ergebnis des Spiels getwittert").

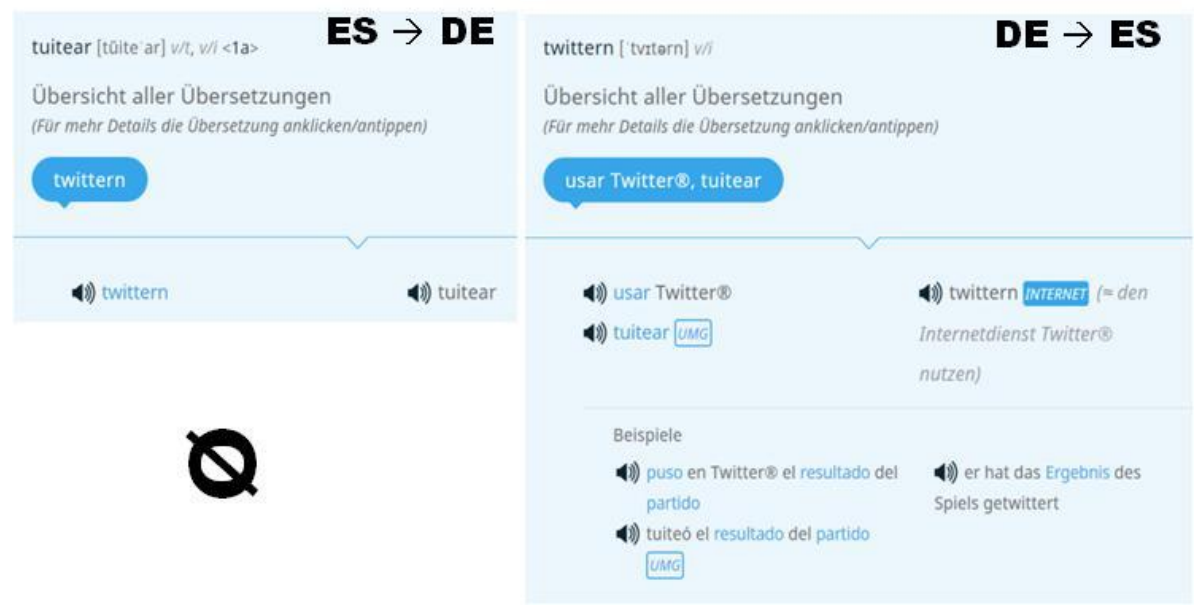

Abb. 18: Bearbeitete Bildschirmausdrucke der Einträge zu tuitear und twittern in Langenscheidt-Online [22.02.18]

Auf Abbildung 19, die die Einträge zu posten/postear illustriert, lässt sich ebenfalls feststellen, dass der Zugriff $\mathrm{zu}$ den Gebrauchsbeispielen ausschließlich über das Deutsche als Ausgangsprache erfolgt. Der spanische Eintrag liefert zwar Informationen zu Transitivität (vgl. , $\left.{ }^{\mathrm{v}} / \mathrm{t}^{\prime \prime}\right)$ und $\mathrm{zu}$ der semantischen bzw. lexikalischen Füllung des Direktobjekts (vgl. „Kommentar"), aber zu den anschaulichen Gebrauchsbeispielen für beide Sprachen gelangt man erst, wenn man auf das deutsche Übersetzungsäquivalent klickt und zum deutschen Eintrag weitergeleitet wird. 


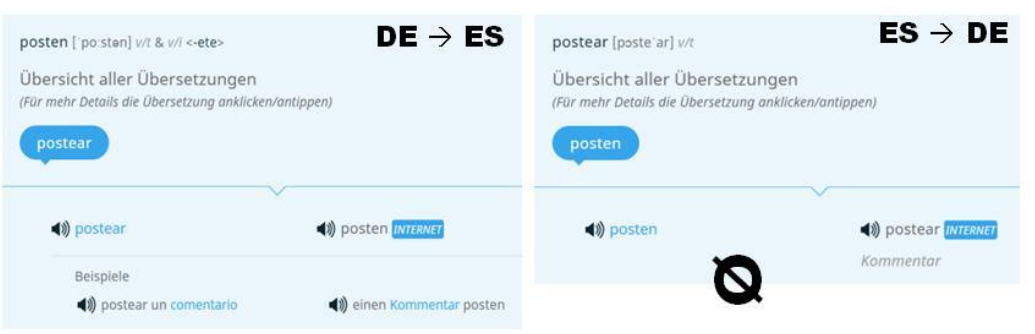

Abb. 19: Bearbeitete Bildschirmausdrucke der Einträge zu posten und postear in Langenscheidt-Online [22.02.18]

Das letzte Beispiel liefert Abbildung 20, die die Einträge zum spanischen wasapear [whatsappen] und deutschen mailen im Vergleich präsentiert. Im Eintrag zu mailen gelangt man nach den Überschrift-Informationen direkt zum Beispielbereich ohne weitere Aufschlüsselung der Entsprechungsvorschläge. Den Beispielen kann der Benutzer relevante Informationen zu Semantik, Syntax und teilweise Gebrauchskontext des Verbs entnehmen. Dies bestätigt die Beobachtung (siehe oben), dass illustrative Beispiele nur in deutschen Einträgen angeboten werden. Der spanische Eintrag $\mathrm{zu}$ wasapear [whatsappen] seinerseits richtet sich eher auf Rezeptionszwecke, da die angebotenen deutschen Entsprechungen bzw. Übersetzungsäquivalente zu wasapear den Status als feste Verbindungen oder typische Verwendungen nicht besitzen. Sie scheinen, nicht konventionalisiert zu sein. Sie können vielmehr als Bedeutungserklärung angesehen werden. Diese Hypothese wird durch weitere Konsultationen bekräftigt. Die betreffenden Ausdrücke "WhatsApp nutzen" und "WhatsApp-Nachrichten verschicken" können weder über das Stichwort "WhatsApp“, „nutzen" oder „verschicken" im Wörterbuch aufgerufen werden. Im Gegensatz dazu gelangt man immer $\mathrm{zu}$ den spanischen Kollokationen mandar/enviar algo a alguien por correo electrónico [jemandem etwas per elektronischer Post schicken/ senden] und mandar/enviar un mail/e-mail a alguien [jemandem eine Mail/E-Mail schicken/senden], die als Entsprechungen zu mailen gelten. Sie sind sowohl unter ihren Nominalkomponenten "correo", "mail" und "e-mail" als auch unter ihren Verbalbasen „mandar" und „enviar“ im Wörterbuch abrufbar.

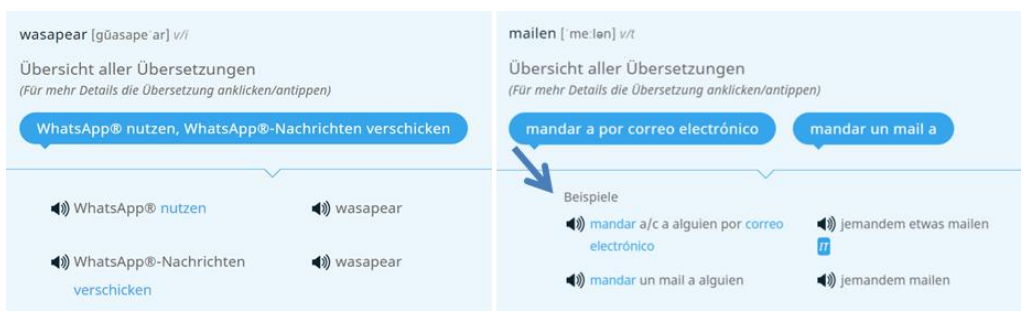

Abb. 20: Bearbeitete Bildschirmausdrucke der Einträge $\mathrm{zu}$ wasapear und mailen aus Langenscheidt-Online im Vergleich [14.03.18] 


\subsection{Schlussfolgerung zu Informationsangebot in bilingualen Online-Wör- terbüchern}

Anhand der neuen MKV des Deutschen und Spanischen ist die Aufführung von Neologismen in drei im Internet angebotenen zweisprachigen Wörterbüchern für das Sprachenpaar Spanisch-Deutsch mit Fokus auf der Produktion in L2 untersucht worden. Das lexikografische Angebot ist auf Adäquatheit und Nützlichkeit bei (a) der Produktion in der Fremdsprache, (b) dem Such-Auffindungsprozess (insbesondere von komplexen Einheiten) und (c) der ausgangsund zielsprachigen Disambiguierung überprüft worden. Nach einer kritischen Beurteilung der Ergebnisse dieser Untersuchung aus kontrastiver Perspektive lassen sich folgende Schlüsse ziehen:

(a) Das Angebot an morphosyntaktischen und lexikosemantischen Daten erweist sich für die richtige bzw. kontextadäquate Anwendung der Ziellemmata in der Fremdsprache in einigen Fällen als unzureichend. Der Benutzer ist oft gezwungen, weitere Konsultationen zu tätigen, um auf die gesuchten Informationen zu gelangen. Ferner ist dieses Informationsangebot manchmal bei den Benutzereinträgen in PONS-Online unvollständig und in Leo.org unsystematisch. In Langenscheidt-Online sind die spanischen und deutschen Einträge mit unterschiedlicher Sorgfältigkeit bzw. Ausführlichkeit ausgearbeitet. Allen drei Wörterbüchern mangelt es an anschaulichen Beispielen, obwohl bestätigt wurde, dass Benutzer einen schnellen Zugriff $\mathrm{zu}$ grammatischen Informationen finden, wenn diese in Beispielen dargestellt werden (vgl. Bogaards und Van der Kloot 2001: 117-118). Die angebotenen Beispiele scheinen Kompetenz- und keine Korpus- bzw. Originalbeispiele zu sein (vgl. Engelberg und Lemnitzer 2009: 236-238, Prinsloo 2013). Ein Vorteil von Kompetenzbeispielen im Vergleich zu Original- bzw. nicht modifizierten Korpusbeispielen ist, dass sie kürzer sind und einfacher zu verstehen sind. Auf der anderen Seite fehlen denen oft kontextuelle Informationen und sie werden als künstlich empfunden (vgl. Prinsloo 2013: 512-513).

(b) Die Auffindung komplexer Einheiten hat sich meistens als erfolgreich bestätigt. Obwohl diese oft erst nach mehreren Konsultationen erfolgte und der gesuchte Ausdruck meistens im Artikeltext verborgen ist. Die Ausnahme bilden die Benutzereinträge in PONS-Online, da die Korrelation dieser Beiträge miteinander und mit anderen Redaktionseinträgen fehlerhaft ist. Die Suche nach komplexen Einheiten hat in den drei Wörterbüchern jedoch nicht immer zu einfachen Lexemen in der Zielsprache (DaF) geführt.

(c) Die angebotenen Daten erlauben - in der Regel - die Ausgangslemmata semantisch adäquat zu disambiguieren. Die syntagmatische und pragmatische Disambiguierung sowie die korrekte Auswahl der Ziellemmata können hingegen in einigen Fällen schwer fallen. Dies ist besonders der Fall in Leo.org, da 
unterschiedliche Lesarten und sogar Homonyme nicht ausreichend gut differenziert sind und gemeinsam in einem einzigen Artikel dargestellt werden.

Zusammenfassend bieten die untersuchten zweisprachigen Wörterbücher zwar morphosyntaktische Daten und Gebrauchsbeispiele, aber dieses Angebot ist für die adäquate Produktion in der Fremdsprache nicht immer behilflich; insbesondere im Falle von Neologismen, da in diesen Einträgen meistens die Bedeutungserläuterung im Fokus steht. Darüber hinaus sind keine relevanten Unterschiede in dem Sprachangebot der drei Wörterbücher festgestellt worden. Bezüglich der Benutzerbeteiligung scheint das semi-kollaborative Wörterbuch von LEO einen höheren Grad an Stabilität und Zuverlässigkeit als das kollaborativ-institutionelle Wörterbuch von PONS aufzuweisen, da bei dem letzteren den Eindruck erweckt wird, dass die Benutzereinträge nicht auf Qualität geprüft sind. Diese werden mit dem Etikett „Benutzereintrag“ versehen, das sie von den anderen Einträgen unterscheidet. Die Benutzereinträge in PONS sind in der Makro- und teilweise in der Mikrostruktur des Wörterbuches integriert, da sie dasselbe Layout wie Redaktionseinträge aufweisen und ebenfalls aufrufbar sind. Sie sind aber in der Mediostruktur nicht integriert, da sie nicht miteinander und mit den anderen Einträgen vernetzt sind. Diese Integration erfolgt erst, wenn sie von der Redaktion überprüft und übernommen werden. Dann sind sie von den Redaktionseinträgen nicht mehr zu unterscheiden.

\section{Exemplarischer Vergleich der lexikografischen Daten mit korpus- basierten Daten}

Auf Grundlage einer Korpusuntersuchung medialer Kommunikationsverben des Deutschen und des Spanischen, die im Rahmen des im Abschnitt 1 erwähnten Dissertationsprojekts durchgeführt worden ist, lässt sich aus empirischen Korpusdaten feststellen, dass gerade Informationen zu dem Gebrauchskontext, dem lexikosyntaktischen Kombinationspotenzial und der Argumentstruktur unter anderen syntagmatischen Informationen besonders wichtig sowohl für die Übersetzung als auch für die freie Textproduktion in der Fremdsprache sind, da die festgestellten Divergenzen zwischen den Verben beider Sprachen genau darin liegen. Pro Verb wurden durchschnittlich 150 Belege aus den entsprechenden Corpora from the Web (COW) für das Deutsche und das Spanische (realise 2012 und 2014) der Freien Universität Berlin (vgl. Schäfer 2015). Zwei ausgewählte Beispiele anhand der Verben posten und twittern sollen zur Illustration dienen.

(i) Das Lokativ-Argument bei posten:

In der betreffenden Korpusuntersuchung ist festgestellt worden, dass das deutsche Verb posten in fast der Hälfte seiner Realisierungen mit einem Lokativ-Argument (LOC) vorkommt (siehe Abb. 21) $)^{8}$. Dieses Argument kann als Adverbial- oder Präpositionalphrase (AP oder PP) realisiert werden. Bezüg- 
lich der präpositionalen Realisierung des Lokativ-Arguments weist das deutsche Verb eine breitere Vielfalt an Präpositionen auf, obwohl eine Dominanz der Präposition in zu erkennen ist.

\begin{tabular}{|c|c|c|c|}
\hline $\begin{array}{l}\text { Lokativ-Argument im } \\
\text { Zusammenhang mit poste }\end{array}$ & $47 \%$ & & \\
\hline $\mathrm{K}_{\mathrm{NP}} \mathrm{V} \mathrm{M}_{\mathrm{NP}} \mathrm{LOC}_{\mathrm{PP} \text { bei }}$ & $1,3 \%$ & \multirow{8}{*}{ 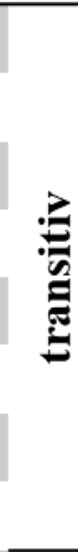 } & \multirow{14}{*}{ 尝 } \\
\hline $\mathrm{K}_{\mathrm{NP}} \mathrm{V} \mathrm{M}_{\mathrm{NP}} \mathrm{LOC}_{\mathrm{PP} \text { unter }}$ & $1 \%$ & & \\
\hline $\mathrm{K}_{\mathrm{NP}} \mathrm{V} \mathrm{M}_{\mathrm{NP}} \mathrm{LOC}_{\mathrm{PP} \text { an }}$ & $1 \%$ & & \\
\hline $\mathrm{K}_{\mathrm{NP}} \mathrm{V} \mathrm{M}_{\mathrm{NP}} \mathbf{L O C}_{\mathbf{P P} \text { in }}$ & $4,5 \%$ & & \\
\hline $\mathrm{K}_{\mathrm{NP}} \mathrm{V} \mathrm{M}_{\mathrm{NP}} \mathbf{L O C}_{\mathrm{PP} \text { in }+ \text { Akk }}$ & $2 \%$ & & \\
\hline $\mathrm{K}_{\mathrm{NP}} \mathrm{V} \mathrm{M}_{\mathrm{NP}} \mathrm{LOC}_{\mathrm{PP} \text { auf }}$ & $2,2 \%$ & & \\
\hline$K_{\mathrm{NP}} V \mathbf{M}_{\mathrm{NP}} \mathbf{L O C}_{\mathrm{AP} \text { hier/da }}$ & $10 \%$ & & \\
\hline 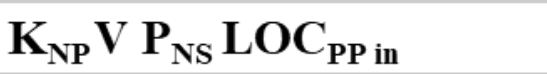 & $9 \%$ & & \\
\hline $\mathrm{K}_{\mathrm{NP}} \mathrm{V} \mathrm{T}_{\mathrm{PP} \text { über }} \mathbf{L O C}_{\mathbf{P P} \text { in }}$ & $0,5 \%$ & \multirow{6}{*}{ 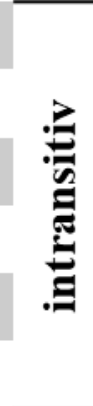 } & \\
\hline $\mathrm{K}_{\mathrm{NP}} \mathrm{VT}_{\mathrm{PP} z \mathrm{u}} \mathbf{L O C}_{\mathbf{P P} \text { in }}$ & $0,5 \%$ & & \\
\hline $\mathrm{K}_{\mathrm{NP}} \mathrm{V} \mathbf{L} \mathbf{O C}_{\mathbf{P P} \text { in }}$ & $5 \%$ & & \\
\hline $\mathrm{K}_{\mathrm{NP}} \mathrm{V} \mathrm{LOC}_{\mathrm{AP} \text { hier }}$ & $4 \%$ & & \\
\hline $\mathrm{K}_{\mathrm{NP}} \mathrm{V} \mathrm{LOC}_{\mathrm{AP} \text { dort }}$ & $0,5 \%$ & & \\
\hline $\mathrm{K}_{\mathrm{NP}} \mathrm{V} \mathrm{LOC}_{\mathrm{AP} \text { hier }}$ FIN umzu & $0,5 \%$ & & \\
\hline $\mathrm{M}_{\mathrm{NP}} \mathrm{V} \mathbf{L} \mathbf{O C}_{\mathbf{P P} \text { in }}$ & $2 \%$ & & \multirow{7}{*}{$\begin{array}{l}\vec{Z} \\
\vdots \\
\vdots \\
\vdots \\
=\end{array}$} \\
\hline $\mathrm{M}_{\mathrm{NP}} \mathrm{V} \mathrm{LOC}_{\mathrm{PP} \text { auf }}$ & $1 \%$ & & \\
\hline $\mathrm{M}_{\mathrm{NP}} \mathrm{V} \mathrm{LOC}_{\mathrm{AP} \text { dort }}$ & $0,5 \%$ & & \\
\hline $\mathrm{M}_{\mathrm{NP}} \mathrm{V} \mathrm{LOC}_{\mathrm{AP} \text { hier }}$ & $1,3 \%$ & & \\
\hline $\mathrm{M}_{\mathrm{NP}} \mathrm{V} \mathrm{LOC}_{\mathrm{AP} \text { hier }} \mathrm{K}_{\mathrm{PP} \text { von }}$ & $0,5 \%$ & & \\
\hline $\mathrm{T}_{\mathrm{PP} \text { über }} \mathrm{V} \mathbf{L} \mathbf{O} \mathbf{C}_{\mathbf{P P} \text { in }}$ & $0,5 \%$ & & \\
\hline $\mathrm{V} \mathrm{LOC}_{\mathrm{PP} \text { in }}$ & $0,5 \%$ & & \\
\hline
\end{tabular}

Abb. 21: Realisierungen von posten, in denen ein Lokativ-Argument vorkommt und ihre Häufigkeit 
Diese Informationen stellen die Relevanz des Lokativ-Arguments bei dem Verben posten heraus. Sie sind jedoch in keinem der konsultierten Wörterbücher (siehe Abschnitt 4. und 5) aufgeführt worden. Die entsprechenden Einträge zu posten enthalten keine Angaben zum Lokativ-Argument. Lediglich aus den "typischen Verwendungen“ im Neologismenwörterbuch des IDS kann man schließen, dass posten ein Lokativ-Argument als Präpositionalphrase realisieren kann (vgl. Eintrag zu posten im Neologismenwörterbuch).

(ii) Realisierung der MESSAGE bei twittern:

Abbildung 22 liefert korpusbasierte Daten zu den Realisierungen von twittern. Das Verb kann sowohl transitiv als auch intransitiv verwendet werden. Das MesSAGE-Argument (M) wird meistens durch eine Nominalphrase (NP) realisiert. Es kann aber auch als Nebensatz ( $\left.P_{\mathrm{NS}}\right)$ oder als Hauptsatz bzw. Zitat $\left(\mathrm{DR}_{\mathrm{HS}}\right)$ kodiert werden. Zudem können MESSAGE- und TOPIK-Argument (T) alternieren. Das Lokativ-Argument zeigt ebenfalls bei den dokumentierten Realisierungen von twittern eine verhältnismäßig starke Präsenz.

In den konsultierten Wörterbüchern sind die Informationen zu Realisierungsmöglichkeiten des MESSAGE-Arguments und zu den anderen möglichen Argumenten von twittern - wenn vorhanden - in den Beispielen verborgen. Lediglich in dem Neologismenwörterbuch des IDS sind etwas expliziter Angaben dazu unter den "typischen Verwendungen“ und bei der Beschreibung der syntaktischen Umgebung des Verbs twittern zu finden (vgl. Eintrag zu twittern im Neologismenwörterbuch). De facto etikettieren es fast alle konsultierten Wörterbücher, die das deutsche Verb twittern inventarisieren, als intransitives Verb.

\begin{tabular}{|c|c|c|c|}
\hline Häufige Realisierungen von twittern & $91 \%$ & \multirow{4}{*}{$\stackrel{\geqq}{:}$} & \multirow{6}{*}{$\frac{Z}{\pi}$} \\
\hline $\mathbf{K}_{\mathrm{NP}} \mathbf{V} \mathbf{M}_{\mathrm{NP}}$ & $20 \%$ & & \\
\hline $\mathrm{K}_{\mathrm{NP}} \mathrm{VDR} \mathrm{DR}_{\mathrm{HS}}$ & $7 \%$ & & \\
\hline $\mathrm{K}_{\mathrm{NP}} \mathrm{VP}_{\mathrm{NS}}$ & $4 \%$ & & \\
\hline $\mathbf{K}_{\mathrm{NP}} \mathbf{V}$ & $22 \%$ & \multirow{2}{*}{ 苞 } & \\
\hline $\mathrm{K}_{\mathrm{NP}} \mathrm{V} \mathrm{T}_{\mathrm{PP} \text { iber/von/zu }}$ & $9 \%$ & & \\
\hline $\mathbf{M}_{\mathrm{NP}} \mathbf{V}$ & $22 \%$ & & \multirow{3}{*}{ 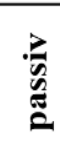 } \\
\hline $\mathrm{M}_{\mathrm{NP}} \mathrm{VLOC}$ AP/PP & $4 \%$ & & \\
\hline $\mathrm{V}$ & $3,5 \%$ & & \\
\hline
\end{tabular}

Abb. 22: Realisierungen von twittern, die häufiger als 3\% sind

Die Befunde bestätigen die Notwendigkeit einer lexikografischen Beschreibung von Neologismen, in der neben ihrer Bedeutung auch ihre syntagmatischen Eigenschaften und ihre kontextuelle Lage u.a. miteinbezogen sind. Dies gewährt bereits das Neologismenwörterbuch des IDS für das Deutsche. In der sprachvergleichenden (Lerner-)Lexikografie hingegen ist momentan ein 
derartiges Werk nicht vorhanden. Die Lernende einer Fremdsprache verstehen aber Neologismen als wichtigen Bestandteil des gängigen Alltagswortschatzes und müssen sie in der Praxis korrekt einsetzen. Die am häufigsten verwendeten zweisprachigen Online-Wörterbücher im spanisch-deutschen Kontext decken diesen Bedarf nur teilweise, da sie in der Regel Benutzerszenarios für die fremdsprachige Rezeption und die Rückübersetzung anvisieren (vgl. Tarp 2008: 147-149 und 161-163, Wiegand et al. 2010: 23-24 und 83-84).

\section{Plädoyer für ein zweisprachiges Neologismenwörterbuch Spanisch $\nleftarrow \rightarrow$ Deutsch}

Die bereits präsentierten Untersuchungsergebnisse zeigen, dass die Erstellung eines zwei- bzw. mehrsprachigen Neologismenwörterbuchs sinnvoll und in dem konkreten Fall für das Sprachenpaar Spanisch $\leftrightarrow$ Deutsch auch notwendig ist. Sie stimmen gleichzeitig mit den Ergebnissen anderer aktueller lexikografischer Studien überein, die auch eine ungenaue Berücksichtigung der möglichen Wörterbuchbenutzungssituationen sowie ein teilweise unvollständiges Informationsangebot bestätigen (vgl. Meliss 2013, 2015a, 2015b, 2016). Davon zeugen ebenfalls vielfältige einschlägigen Benutzerumfragen (vgl. Domínguez Vázquez et al. 2013, Meliss 2015b, Fernández Méndez et al. 2016), in denen die Benutzer selbst behaupten, einige Informationen vermisst $\mathrm{zu}$ haben, die vor allem das syntagmatische Umfeld und die Kombinatorik des gesuchten Wortes betreffen (vgl. Meliss 2015b: 420-421).

\section{Danksagung}

Ich möchte mich bei Danie Prinsloo für Ihre unendliche Geduld und die Unterstützung bei der Überarbeitung dieses Artikels bedanken. Ebenfalls bedanke ich mich bei den beiden anonymen Gutachtern für Ihre hilfreiche Kommentare.

\section{Endnoten}

1. In der vorliegenden Studie wird der allgemeine Begriff „Benutzer,, (vgl. Wiegand et al. 2010: 675) sowohl zur Bezeichnung des potenziellen Benutzers (Wiegand 1998: 504, Tarp 2008: 54-55) als auch des Benutzers-in-actu (Wiegand et al. 2010: 678) in den konkreten Beispielen verwendet. Außerdem haltet es sich immer um einen fremdsprachigen Benutzer, da zumindest ein Teil der Wörterbuchbenutzung in einer Fremdsprache erfolgt (vgl. Wiegand et al. 2017: 341). Ferner soll nochmal betont werden, dass sich die hier ausgewählte generische Form des Maskulinums sowohl auf männliche Benutzer und Benutzerinnen als auch auf Wörterbuchbenutzer bezieht, die sich mit keinem beider sozialen Geschlechter identifizieren. Die Variante "die Wörterbuch benutzende Person“ bleibt ausgeschlossen, da sie sich lediglich auf den Benutzer-in-actu beschränkt (vgl. Wiegand 1998: 500-501). 
2. Insbesondere hinsichtlich des Print-Formats sollte man die bei den Neologismenwörterbüchern problematische Aktualitätsfrage berücksichtigen (vgl. Engelberg und Lemnitzer 2009: 58-60).

3. Da die betreffenden Wörterbücher in Bezug auf die Bedürfnisse ihres potenziellen Benutzers (vgl. Tarp 2008: 54-58), d.h. in Bezug auf den Benutzungsanlass und -grund (Wiegand et al. 2010: 681 und 685) beschrieben worden sind, eignet sich die angeführte Terminologie der an dem lexikografischen Institut der Universität Aarhus entwickelten Function Theory (vgl. Tarp 2008) insbesondere, denn in diesem theoretischen Rahmen der potenzielle Benutzer eine zentrale Rolle spielt. Zur Diskussion über die relevantesten Unterschiede zwischen der Function Theory und der weitverbreiteten lexikografischen Theorie von Wiegand (1998) siehe Tarp 2008: 39-43 und 80-97.

4. Die Printversion des Wörterbuches, die die Neologismen der Nuller- und Zehnerjahren bis 2010 erfasst (vgl. Steffens und Al-Waldi 2015) wurde für die Analyse nicht berücksichtigt, weil die Online-Version inhaltlich darüber hinaus geht und dazu die neuen Wörter der letzten Jahre aufführt.

5. Siehe Engelberg und Lemnitzer (2009: 99-112) für allgemeine Informationen zum Thema Suche in Online-Wörterbüchern.

6. Letzte Konsultation wurde am 06.03.2018 getätigt.

7. Ein Vorteil kollaborativer Benutzerbeteiligung ist gerade die Schließung inhaltlicher Lücken; insbesondere bezüglich Neologismen, Fachsprache und dialektaler Varietäten (vgl. Abel und Meyer 2016: 278-280). Dennoch sind alle in den kollaborativen Wörterbüchern inventarisierten MKV auch in dem institutionellen Wörterbuch Langenscheidt abrufbar (siehe Abb. 8).

8. Erklärung zu Abkürzungen für die Argumentenbeschreibung: $\mathrm{K}=$ Kommunikator, $\mathrm{M}=$ Message, $\mathrm{DR}=$ direkte Rede, $\mathrm{P}=$ Proposition, $\mathrm{T}=$ Topik, $\mathrm{LOC}=$ interner Lokativ, FIN = Finalität, $\mathrm{V}=$ Verb, $\mathrm{NP}=$ Nominalphrase, $\mathrm{AP}=$ Adverbialphrase, $\mathrm{PP}=$ Präpositionalphrase, HS $=$ Hauptsatz, NS= Nebensatz.

\section{Primäre und sekundäre Quellen}

Abel, A. und C.M. Meyer. 2016. Nutzerbeteiligung. Klosa, A. und C. Müller-Spitzer (Hrsg.). 2016: 249-290.

Bergenholtz, H. 2011. Access to and Presentation of Needs-Adapted Data in Monofuncional Internet Dictionaries. Fuertes-Olivera, P.A. und H. Bergenholtz (Hrsg.). 2011: 30-53.

Bergenholtz, H. 2012. Concepts for Monofunctional Accounting Dictionaries. Terminology 18(2): 243-263.

Bergenholtz, H. und I. Bergenholtz. 2011. A Dictionary is a Tool, a Good Dictionary is a Monofunctional Tool. Fuertes-Olivera, P.A. und H. Bergenholtz (Hrsg.). 2011: 187-207.

Bogaards, P. und W.A. van der Kloot. 2001. The Use of Grammatical Information in Learners' Dictionaries. International Journal of Lexicography 14(2): 97-121.

COW: Corpora from the Web. http://corporafromtheweb.org/.

Debus-Gregor, E. und U. Heid. 2013. Design Criteria and 'Added Value' of Electronic Dictionaries for Human Users. Gouws, R.H. et al. (Hrsg.). 2013: 1001-1013.

DeReKo: Das Deutsche Referenzkorpus. http://www1.ids-mannheim.de/kl/projekte/korpora/.

Die Wortwarte. http://www.wortwarte.de/. 
Domínguez Vázquez, M.J. (Hrsg.). 2013. Trends in der deutsch-spanischen Lexikographie. Frankfurt a.M.: Peter Lang.

Domínguez Vázquez, M.J., M. Mirazo Balsa und V. Vidal Pérez. 2013. Wörterbuchbenutzung: Erwartungen und Bedürfnisse. Ergebnisse einer Umfrage bei Deutsch lernenden Hispanophonen. Domínguez Vázquez, M.J. (Hrsg.). 2013: 135-172.

Dou, X. 2004. Deutsch-chinesisches Neologismenwörterbuch. De-Han xinci cidian. Beijing: The Commercial Press.

Engelberg, S. und L. Lemnitzer. 2009. Lexikographie und Wörterbuchbenutzung. Tübingen: Stauffenburg.

Engelberg, S. und C. Müller-Spitzer. 2013. Dictionary Portals. Gouws, R.H. et al. (Hrsg.). 2013: 1023-1035.

Engelberg, S. und A. Storrer. 2016. Typologie von Internetwörterbüchern und -portalen. Klosa, A. und C. Müller-Spitzer (Hrsg.). 2016: 31-63.

Fernández Méndez, M., M. Franco Barros und I. Sueiro Orallo. 2016. El uso de los diccionarios de/ para ELE y DaF: análisis de una encuesta desde una perspectiva contrastiva. Robles i Sabater, F., D. Reimann und R. Sánchez Prieto (Hrsg.). 2016. Sprachdidaktik Spanisch-Deutsch: Forschungen an der Schnittstelle von Linguistik und Fremdsprachendidaktik: 73-92. Romanistische Fremdsprachenforschung und Unterrichtsentwicklung 6. Tübingen: Narr Francke Attempto.

Fuertes-Olivera, P.A. 2009. The Function Theory of Lexicography and Electronic Dictionaries: WIKTIONARY as a Prototype of Collective Free Multiple-Language Internet Dictionary. Bergenholtz, H., S. Nielsen und S. Tarp (Hrsg.). 2009. Lexicography at a Crossroads. Dictionaries and Encyclopedias Today, Lexicographical Tools Tomorrow: 99-134. Linguistic Insights: Studies in Language and Communication 90. Bern: Peter Lang.

Fuertes-Olivera, P.A. und H. Bergenholtz (Hrsg.). 2011. e-Lexicography: The Internet, Digital Initiatives and Lexicography. London/New York: Continuum.

Gierden Vega, C., B. Eggelte, B. Heinsch und D. Hofmann. 2010. Compendio temático de neologismos Alemán - Español: deutsche Neubildungen. Stuttgart: Ibidem.

González Ribao, V. 2019. Das Zusammenspiel von Verb- und Musterbedeutung der medialen Kommunikationsverben im Sprachvergleich Deutsch-Spanisch-Englisch. Eine konzeptuell orientierte lexikologische Studie. Unv. Diss. Santiago de Compostela: Universität Santiago de Compostela, Spanien. Online: http://hdl.handle.net/10347/18728.

Gouws, R.H., U. Heid, W. Schweickard und H.E. Wiegand (Hrsg.). 2013. Dictionaries. An International Encyclopedia of Lexicography. Supplementary Volume: Recent Developments with Focus on Electronic and Computational Lexicography. Handbücher zur Sprach- und Kommunikationswissenschaft 5.4. Berlin/Boston: De Gruyter Mouton.

Heid, U. 2011. Electronic Dictionaries as Tools: Towards an Assessment of Usability. Fuertes-Olivera, P.A. und H. Bergenholtz (Hrsg.). 2011: 287-304.

Herbst, T. 2009. Item-specific Syntagmatic Relations in Dictionaries. Nielsen, S. und S. Tarp (Hrsg.). 2009: 281-308.

Klosa, A. und C. Müller-Spitzer (Hrsg.). 2016. Internetlexikographie. Ein Kompendium. Berlin/Boston: De Gruyter.

Langenscheidt: Online-Angebot Spanisch $\leftrightarrow$ Deutsch. https://de.langenscheidt.com/deutsch-spanisch/.

Lemnitzer, L. 2010. Neologismenlexikographie und das Internet. Lexicographica 26: 65-78.

LEO: Online-Wörterbuch Spanisch $\leftarrow \rightarrow$ Deutsch. https://www.leo.org/alem\%C3\%A1n-espa\%C3\%B1ol. 
Lew, R. 2011. Online Dictionaries of English. Fuertes-Olivera, P.A. und H. Bergenholtz (Hrsg.). 2011: 230-250.

Meliss, M. 2013. Das zweisprachige Wörterbuch im bilateralen deutsch-spanischen Kontext. Alte und neue Wege. Domínguez Vázquez, M.J. (Hrsg.). 2013: 61-87.

Meliss, M. 2015a. Zum Kombinationspotenzial von Verben in einsprachigen DaF-Lernerwörterbüchern - kritische Bestandsaufnahme und neue Anforderungen. Zeitschrift für Deutsch als Fremdsprache 1(2015): 14-27.

Meliss, M. 2015b. Was suchen und finden Lerner des Deutschen als Fremdsprache in aktuellen Wörterbüchern? Auswertung einer Umfrage und Anforderungen an eine aktuelle DaFLernerlexikographie. Roelcke, T. (Hrsg.). 2015. Wörterbücher für Deutsch als Fremdsprache Probleme und Perspektiven: 401-432. Info Daf-Themenheft 42(4).

Meliss, M. 2016. Wie viele und welche bilingualen Online-Wörterbücher brauchen wir für den DaF-Bereich? Erstellung eines Kriterienrasters und erste Bestandsaufnahme aus der Sicht eines hispanophonen Lernerkontextes. Cuartero Otal, J., J.P. Larreta Zulategui und C. Ehlers (Hrsg.). 2016. Querschnitt durch die deutsche Sprache aus spanischer Sicht. Perspektiven der kontrastiven Linguistik: 187-210. Sprachwissenschaft 30. Berlin: Frank \& Timme.

Müller-Spitzer, C. 2010. OWID - A Dictionary Net for Corpus-Based Lexicography of Contemporary German. Dykstra, A. und T. Schoonheim (Hrsg.). 2010. Proceedings of the XIV Euralex International Congress, Leeuwarden, 6-10 July 2010: 445-452. Leeuwarden: Fryske Akademy.

Neologismenwörterbuch. http://www.owid.de/wb/neo/start.html.

Nielsen, S. und S. Tarp (Hrsg.). 2009. Lexicography in the 21st Century. In Honour of Henning Bergenholtz. Terminology and Lexicography Research and Practice 12. Amsterdam/Philadelphia: John Benjamins.

PONS: Online-Wörterbuch Spanisch $\leftrightarrow$ Deutsch. https://de.pons.com/.

Prinsloo, D.J. 2013. New Developments in the Selection of Examples. Gouws et al. (Hrsg.). 2013: 509-516

Quasthoff, U. (Hrsg.). 2007. Deutsches Neologismenwörterbuch. Berlin/New York: De Gruyter.

Samaniego Fernández, E. und B. Pérez Cabello de Alba. 2011. Conclusions: Ten Key Issues in e-Lexicography for the Future. Fuertes-Olivera, P.A. und H. Bergenholtz (Hrsg.). 2011: 305-311.

Schäfer, R. 2015. Processing and Querying Large Web Corpora with the COW14 Architecture. Bański, P., H. Biber, E. Breiteneder, M. Kupietz, H. Lüngen und A. Witt (Hrsg.). 2015. Proceedings of the 3rd Workshop on Challenges in the Management of Large Corpora (CMLC-3): 28-34. Mannheim: Institut für Deutsche Sprache.

Scheller-Boltz, D. und H. Weinberger. 2017. Lexikografie und Innovation. Zur Vielfältigkeit in der gegenwärtigen Lexikografie und warum wir Lexikografie heute anders verstehen müssen. Scheller-Boltz, D. und H. Weinberger (Hrsg.). 2017. Lexikografische Innovation. Innovative Lexikografie. Bi- und multilinguale Wörterbücher in Gegenwart und Zukunft: Projekte, Konzepte, Visionen: 7-18. Hildesheim: Olms.

Steffens, D. und D. Al-Waldi. ${ }^{32015}$. Neuer Wortschatz: Neologismen im Deutschen 2001-2010. Mannheim: Institut für Deutsche Sprache.

Steffens, D. und O. Nikitina. 22016. Deutsch-russisches Neologismenwörterbuch. Neuer Wortschatz im Deutschen 1991-2010. Немеико-русский словарь неологизмов. Новая лексика в немеиком языке 1991-2010. Mannheim: Institut für Deutsche Sprache. Online: http://www.owid.de/drnwb/ [08.2018] 
Tarp, S. 2008. Lexicography in the Borderland between Knowledge and Non-Knowledge. General Lexicographical Theory with Particular Focus on Learner's Lexicography. Lexicographica. Series Maior 134. Tübingen: Max Niemeyer.

Tarp, S. 2009. Reflections on Data Access in Lexicographic Works. Nielsen, S. und S. Tarp (Hrsg.). 2009: 43-62.

Tarp, S. 2013a. New Developments in Learners' Dictionaries III: Bilingual Learners' Dictionaries. Gouws, R.H. et al. (Hrsg.). 2013: 425-431.

Tarp, S. 2013b. Lexicographic Functions. Gouws, R.H. et al. (Hrsg.). 2013: 460-468.

Verlinde, S. 2011. Modelling Interactive Reading, Translation and Writing Assistants. Fuertes-Olivera, P.A. und H. Bergenholtz (Hrsg.). 2011: 275-286.

Wiegand, H.E. 1998. Wörterbuchforschung: Untersuchungen zur Wörterbuchbenutzung, zur Theorie, Geschichte, Kritik und Automatisierung der Lexikographie. 1. Teilband. Berlin/New York: De Gruyter.

Wiegand, H.E., M. Beißwenger, R.H. Gouws, M. Kammerer, A. Storrer und W. Wolski (Hrsg.). 2010. Wörterbuch zur Lexikographie und Wörterbuchforschung. Dictionary of Lexicography and Dictionary Research. Band/Volume 1: Systematische Einführung/Systematic Introduction A-C. Berlin/New York: De Gruyter.

Wiegand, H.E., M. Beißwenger, R.H. Gouws, M. Kammerer, A. Storrer und W. Wolski (Hrsg.). 2017. Wörterbuch zur Lexikographie und Wörterbuchforschung. Dictionary of Lexicography and Dictionary Research. Band/Volume 2: D-H. Berlin/New York: De Gruyter.

Worbs, E., A. Markowski und A. Meger. 2007. Polnisch-deutsches Wörterbuch der Neologismen. Neuer polnischer Wortschatz nach 1989. Wiesbaden: Harrassowitz. 